\title{
1 Feedback inhibition and its control in an insect olfactory circuit
}

2

3 Authors: Subhasis Ray, Zane N. Aldworth and Mark A. Stopfer ${ }^{1}$

4 Author affiliation: Section on Sensory Coding and Neural Ensembles, NICHD, NIH,

5 Bethesda, Maryland 20892 USA

\section{Abstract}

$7 \quad$ Inhibitory neurons play critical roles in regulating and shaping olfactory responses in

8 vertebrates and invertebrates. In insects, these roles are performed by relatively few

9 neurons, which can be interrogated efficiently, revealing fundamental principles of olfactory coding. Here, with electrophysiological recordings from the locust and a large-scale biophysical model, we analyzed the properties and functions of GGN, a unique giant GABAergic neuron that plays a central role in structuring olfactory codes in the locust mushroom body. Analysis of our in vivo recordings and simulations of our model of the olfactory network suggests that GGN extends the dynamic range of KCs, and leads us to predict the existence of a yet undiscovered olfactory pathway. Our analysis of GGN's intrinsic properties, inputs, and outputs, in vivo and in silico, reveals basic new features of this critical neuron and the olfactory network that surrounds it.

\section{Introduction}

Olfactory information is transformed dramatically as it travels from the periphery to higher order brain centers. Multiple types of olfactory receptor neurons may respond to a given odor with vigorous bursts of action potentials, while neurons deeper in the brain, in the pyriform cortex (vertebrates) or mushroom body (insects), may respond to the same odor with only a

\footnotetext{
${ }^{1}$ Correspondence: stopferm@mail.nih.gov
} 
spike or two (Laurent \& Naraghi, 1994; Friedrich \& Laurent, 2001; Perez-Orive et al., 2002;

Cang \& Isaacson, 2003; Bathellier et al., 2008; Poo \& Isaacson, 2009). In these higher order neurons, information about odors is represented sparsely by the identities of the active neurons (population coding) and in the timing of the few spikes elicited in those neurons (temporal coding)(Perez-Orive et al., 2002; Poo \& Isaacson, 2009; Stettler \& Axel, 2009; Gupta \& Stopfer, 2014). Many studies in vertebrates and invertebrates suggest that multiple mechanisms interact to mediate these transformations, including important inhibitory contributions from GABAergic neurons(Luna \& Pettit, 2010; Papadopoulou et al., 2011; Lin et al., 2014; Palmer \& Harvey, 2014; Large et al., 2016; Large et al., 2018). Here, with intracellular recordings and a new large-scale biophysical model that includes tens of thousands of neurons and spans multiple layers of olfactory processing, we focus on a singularly important inhibitory neuron to investigate the roles of input activity and feedback inhibition in creating a sparse spatio-temporal odor representation in a higher order brain center. Together, our recordings and models point to new functions, neural connectivity patterns, and mechanisms that underlie transformations in the format of olfactory information.

The locust olfactory system is tractable owing to its relative simplicity and is well studied. At rest, olfactory receptor neurons (ORNs) are spontaneously active, evoking activity in the antennal lobe's projection neurons (PNs; Figure 1a)(Joseph et al., 2012). Odorants can elicit increases or decreases in the firing rates of ORNs, and patterns of spikes that can include periods of excitation and inhibition that vary with the odor. The heterogeneous responses of ORNs drive firing patterns in PNs that are further shaped by inhibition from the antennal lobe's local interneurons (LN)(Raman et al., 2010). Spikes in PNs are also coaxed into rhythmic waves by fast reciprocal interactions between excitatory PNs and inhibitory LNs(MacLeod \& Laurent, 1996; Bazhenov et al., 2001). Odor-elicited firing patterns distributed across the population of PNs are informative about the identity, concentration, and timing of the odor(Laurent et al., 1996; Stopfer et al., 2003; Brown et al., 2005). This 
information is carried by PNs to the mushroom body and the lateral horn. Within the mushroom body, the primary neurons are Kenyon cells (KCs). Unlike the volubly spiking PNs, the KCs are nearly silent at rest and respond very selectively to odors with very few spikes(Laurent \& Naraghi, 1994; Perez-Orive et al., 2002; Joseph et al., 2012). Thus, any given odor evokes responses in a small fraction of the $\mathrm{KC}$ population, and any $\mathrm{KC}$ responds to a small set of odors(Perez-Orive et al., 2002; Stopfer et al., 2003). This sparseness of activity in KCs is thought to arise mainly from two factors: specialized membrane conductances that imbue them with high firing thresholds; and a feedback circuit that tamps down their spiking with cyclic inhibition(Perez-Orive et al., 2002; Demmer \& Kloppenburg, 2009; Papadopoulou et al., 2011; Lin et al., 2014). In the locust the main sources of this inhibition are the giant GABAergic neurons (GGNs), one on each side of the brain(Leitch \& Laurent, 1996; Papadopoulou et al., 2011; Gupta \& Stopfer, 2012).

GGN spans much of each brain hemisphere and branches very widely (Figure 1a). It is reported to receive excitatory input from all 50,000 KCs at synapses within the mushroom body's $\alpha$ lobe and, in turn, provide inhibitory feedback to all KCs $400-500 \mu \mathrm{m}$ away within the calyx. In addition, GGN receives inhibitory input from a spiking neuron aptly named "Inhibitor of GGN" (IG) which itself receives inhibition from GGN (Figure 1a, right)(Papadopoulou et al., 2011). GGN is a non-spiking interneuron. Odor presentations, spiking in KCs, and intracellular current injections have all been shown to depolarize GGN, but none of these stimuli causes GGN to generate spikes; even large depolarizations induced by strong intracellular current injections lead only to passive depolarizing responses(Leitch \& Laurent, 1996; Papadopoulou et al., 2011); and our own observations).

GGN's structure is likely an important factor in its function. GGN is very large, and along its path from the $\alpha$ lobe to the calyx, its initially thick processes divide at myriad branch points into vanishingly thin fibers. Cable theory applied to neurons(Rall, 1964) predicts that a passive electrical signal within such a structure will attenuate dramatically as it encounters cytosolic resistance along the neurites, will attenuate further as it divides at the neuronal 
arbor's branch points, and will leak out through ionic channels in the cell membrane. Prior studies in invertebrates have shown that 2-5 $\mathrm{mV}$ depolarizations in nonspiking interneurons can evoke changes in the membrane potentials of their post-synaptic neurons (Burrows \& Siegler, 1978; Manor et al., 1997). If signals originating in GGN's $\alpha$ lobe attenuate to the extent that they cannot effectively hyperpolarize KCs, then GGN must be exerting its influence on KCs through a different mechanism, perhaps, for example, by inhibiting KCs through local interactions entirely within the mushroom body calyx. To test these ideas, we developed a realistic computational model of GGN to characterize signal attenuation throughout its structure. Our model showed that, although electrical signals undergo substantial attenuation as they travel through GGN, signals arising in its $\alpha$ lobe branches appear to remain strong enough to provide global inhibition to KCs in the calyx.

To further understand the network determinants of GGN's responses to odors, we recorded from it in vivo while delivering a variety of odors to the animal, and then used our large-scale model to investigate the types of network activity needed to generate these patterns. We identified two novel features in the olfactory network. First, to generate the types of membrane potential patterns we observed in GGN, the synaptic connection strengths onto KCs must be heterogeneous. Second, our in vivo recordings of GGN revealed novel, complex response patterns not previously documented, including periods of hyperpolarization, that vary with the odorant. Although GGN receives reciprocal feedback from IG(Papadopoulou et al., 2011), the periods of hyperpolarization could not be explained by disinhibition of IG from GGN. Instead, our model predicts that this behavior could arise if, in addition to receiving input from GGN, IG also receives direct excitation from another, unknown odor-activated pathway. Additionally, our model replicated emergent features of the olfactory network not explicitly programmed into it, such as the appearance of a small portion of KCs spiking at relatively high rates. modeling provide a more complete understanding of how different parts of the olfactory 
system interact. To generate odor-specific temporally patterned responses in GGN and in the mushroom body, temporally-patterned odor evoked excitation from PNs, feedback inhibition from GGN, and inhibition of GGN by odor-driven IG must all cooperate.

\section{Results}

A valuable use of computational modeling is to answer questions about biological systems when those systems are too large, complex, or difficult to study with the tools of physiology alone. Earlier computational studies of the insect olfactory system used relatively simple models of neurons such as integrate and fire or map-based models that collapse entire neuronal structures into a single point(Perez-Orive et al., 2004; Papadopoulou et al., 2011;

114 Arena et al., 2015; Kee et al., 2015; Peng \& Chittka, 2017). However, GGN's giant size, elaborate branching, and passive membrane properties raised questions about its function that could only be addressed by considering properties determined by its morphology. Thus, to understand how the size and shape of GGN affects electrical signal propagation, we constructed a detailed morphological model (available at neuromorpho.org).

To reconstruct the morphology of GGN, we first made intracellular recordings from it in vivo, filled it with dye, and obtained 3D confocal images of the dye-filled cell (Figure 1a, left; Video S1). As previously shown, GGN has a reliable and unique location and morphology(Leitch \& Laurent, 1996; Papadopoulou et al., 2011; Gupta \& Stopfer, 2012). On each side of the brain, its soma is located ventrally, just anterior to the optic nerve. A single neurite emerges from GGN's soma, travels toward the posterior and dorsal side of the brain, and splits there into two branches, one innervating the $\alpha$ lobe and the other the mushroom body. Extending outward, these branching neurites expand in width, becoming much thicker than the primary neurite. The mushroom body branch further divides into two thick processes that innervate the medial and the lateral calyx. A thin neurite emerging from the lateral calyceal branch loops back to the lateral horn, close to the soma, and splits there into many branches. We 
further observed, for the first time, myriad thin fibers that emerge from the stems of the calyceal branches, splitting into very fine feather-like neurites that wrap densely around the

132 peduncle, with some investing the peduncle core (Figure 1b). The neurites in the calyx and 133 lateral horn are dotted with many irregular bouton-like structures (Figure 1c) whereas the branches in $\alpha$ lobe are relatively smooth (Figure $1 d$ ).

In two animals we traced and reconstructed the morphology of GGN from confocal image stacks (Figure 1e). Analyzing these traces, we found that the maximum path length of the neuronal trees (i.e., the maximum distance between any two points on the neuronal tree when traversed along the neurites) was on the order of $2 \mathrm{~mm}$, and the maximum physical length (i.e., Euclidean distance between any two points on the neuron in three-dimensions) was on the order of $1 \mathrm{~mm}$. Some neurites at their thickest were nearly $20 \mu \mathrm{m}$ in diameter.

141 Total traced branch length (i.e., the sum of the lengths of all the neurites) was about $65 \mathrm{~mm}$

142 (although many vanishingly thin branches were too fine to trace). Compared to 96,831

143 vertebrate and invertebrate neurons cataloged in the neuromorpho.org database, GGN fell

144 into the $99.75^{\text {th }}$ percentile for total branch length, and the $99.95^{\text {th }}$ percentile for number of

145 branch points. It is a really big neuron.

\section{Signals travelling through GGN's arbors attenuate significantly}

147 To investigate GGN's electrical properties we constructed passive electrical model cells by

148 transferring the morphology tracings of the two GGNs into the NEURON

149 simulator(Carnevale \& Hines, 2006). Simulation of both models produced qualitatively similar 150 results; the model we describe here was derived from the second neuron we traced because

151 it was imaged at higher resolution. To account for branches and changes in the diameters of

152 processes that affect electrotonic distance, we segmented the model GGN into 5,283

153 compartments. We set the membrane resistivity in the model to $33 \mathrm{k} \Omega-\mathrm{cm}^{2}$ based on

154 published data obtained from other non-spiking neurons in the locust(Laurent, 1991), the

155 specific membrane capacitance to $1 \mu \mathrm{F} / \mathrm{cm}^{2}$, the approximate value for cell 
membranes(Curtis \& Cole, 1938; Hodgkin \& Huxley, 1952; Gentet et al., 2000), and the

157 cytoplasmic resistivity to $100 \Omega$-cm, a typical order-of-magnitude value for neurons(Hodgkin

158 \& Rushton, 1946; Stuart \& Spruston, 1998; Roth \& Häusser, 2001).

159 Feedback signals are thought to travel passively from GGN's $\alpha$ lobe branch to its calyceal branches. To test the extent of passive signal attenuation through GGN's structure, we first simulated voltage clamping the base of the $\alpha$ lobe branch of the GGN model (Figure 2a). Intracellular recordings((Papadopoulou et al., 2011); and our own) show GGN's membrane potential rests at about $-51 \mathrm{mV}$. Because strong odor stimuli depolarize $\mathrm{GGN}$ by about 10 $\mathrm{mV}$ in recordings made near the base of the $\alpha$ lobe branch(Papadopoulou et al., 2011) (also see Figure 4) we first stepped the clamp voltage to $-40 \mathrm{mV}$, and, after holding it there for 450 ms, measured the resulting depolarizations throughout the model GGN (Figure 2a inset). Notably, the extent of signal attenuation was substantial and varied throughout the calyx with depolarizations ranging from $\sim 5-9 \mathrm{mV}$. The signal decreased with branch distance from the $\alpha$ lobe, leaving the least amount of signal at the medial portion of the lateral calyceal branch (Figure 2b, c, Video S2).

171 Since excitatory input typically arrives in GGN not at one point, but rather from many KCs,

172 we then tested a more realistic form of simulated input to the $\alpha$ lobe arbor of GGN by

173 providing nonhomogeneous Poisson spike trains through 500 excitatory model synapses;

174 each synapse had a maximum rate of 20 spikes/s that ramped down linearly to 0 over a 500

175 ms interval (Figure 2d, e). This stimulus set was calibrated to generate a peak depolarization

176 in the thick branches of GGN in the same range we observed in vivo. This test also revealed 177 significant attenuation of voltage in the neuron's distant branches (Figure 2e, f) as well as 178 delays in signal propagation (Figure 2 - figure supplement 1 ).

179 For GGN, neither membrane resistivity (RM) nor cytoplasmic (axial) resistivity (RA) has been measured definitively; yet, for a given morphology, these two parameters determine signal attenuation. Thus, we explored a range of values for these two parameters with the voltage 
clamp simulation approach shown in Figure $2 \mathrm{a}$. We based the RM value range on data obtained from many types of neurons provided by the neuroelectro.org database. For RA, neurophysiological data is sparse, so we explored broadly around the range of published values(Hodgkin \& Rushton, 1946; Stuart \& Spruston, 1998; Roth \& Häusser, 2001). As expected, higher RA yielded greater signal attenuation, whereas higher RM yielded less signal attenuation (Figure $2 \mathrm{~g}$ ). This analysis showed that signal transmission in GGN is robust: except for the most extreme values of this parameter range, signals from the $\alpha$ lobe remained strong enough to support synaptic transmission in the calyx. The extent of depolarization throughout GGN's calyceal arbor varied with location, as quantified in the extended lower lobe in the violin plots in Figure 2c, $\mathrm{f}$ and $\mathrm{g}$.

Branches of GGN receiving weaker signals would be expected to provide less inhibition to their postsynaptic KCs. In a simplified model in which all KCs were strongly stimulated by identical input from PNs, the amount of KC spiking was indeed negatively correlated with local GGN voltage deflections (Figure 2 - figure supplement 2). However, in a more realistic model of the mushroom body network including variable excitatory input from PNs and variable strengths of inhibitory synapses between GGN and KCs, we found the negative correlation between depolarizations measured at presynaptic locations throughout GGN and postsynaptic KC activity was small, and likely negligible (Figure 2 - figure supplement 3 ). This suggests GGN's inhibitory output has a surprisingly uniform influence across the population of KCs regardless of location.

\section{Feedback inhibition expands the dynamic range of $\mathrm{KCs}$}

We used our model to test other basic properties of GGN and the olfactory network 
209 feedback inhibition from APL, the analog of GGN, expands the dynamic range of KCs

210 (Inada, Tsuchimoto, \& Kazama, 2017). Whether feedback inhibition from GGN has a similar

211 effect on KCs is unknown. To test this, we extended our model to include, for simplicity, a

212 single KC receiving feedback inhibition from GGN (Figure 3a). To simulate the KC in this test

213 we used a single compartmental model with Hodgkin-Huxley type ion channels(Wüstenberg

214 et al., 2004). Since a single KC would have negligible effect on GGN, we applied its spiking output to GGN's $\alpha$ lobe branch via 50,000 synapses. Moreover, to avoid unrealistic, strong synchronous input to GGN, we jittered the incoming spike times by applying random synaptic delays between 0 and $60 \mathrm{~ms}$. Thus, after each spike generated by the model KC,

218 GGN received 50,000 EPSPs spread over a 60ms time window. We drove the KC model with a range of tonic current injections and compared its responses to those of an isolated $\mathrm{KC}$ model receiving the same input without feedback inhibition. As expected, baseline inhibition from spontaneous activity in GGN increased the KC's threshold for spiking. Notably, though, the GGN-coupled KC continued to spike over a much larger range of current injection than the isolated $\mathrm{KC}$, which quickly saturated to a level where it could no longer spike (Figure 3b, c). A similar result was obtained when we tested the KC by applying simulated GGN inhibition from a model of the olfactory network described later (Figure 3 figure supplement 1). These results suggested that feedback inhibition from GGN allows an individual KC to function effectively over a larger dynamic range of inputs. In control system analysis, dynamic range is often characterized by the slope of the input-response curve; expanding the dynamic range makes the slope shallower, as we observed in our model

230 (Figures 3c and d; see Methods for the slope calculation).

231 To test this result in vivo, we made simultaneous intracellular recordings from GGN and KCs. We counted the number of spikes elicited in single KCs by small or large amplitude intracellular current pulses in the presence or absence of a simultaneous small or large 234 amplitude depolarizing current injection to GGN (Figure 3e). In 5 of 5 tested KCs $(p<0.033$, binomial test), the slope of the input-response curve with increasing current injection became 
shallower when GGN was depolarized, as predicted by the model (Figure 3f-g),

demonstrating that, in vivo, inhibition from GGN increases the dynamic range of KCs.

238 GGN responses can be complex, including hyperpolarization

239 Our recordings made in vivo from GGN frequently revealed depolarizations lasting

240 throughout an odor presentation, often with additionally depolarizing peaks corresponding to

241 the onset and offset of the odor (Figure 4, Animal 1, hexanol) (see also (Papadopoulou et al., 2011)). Notably, our recordings from GGN also revealed more complex odor-elicited responses than previously reported, including combinations of depolarization and hyperpolarization (Figure 4, Animal 1, hexanal). Moreover, we found that the same GGN could respond differently when different odors were presented; for example, GGNs from Animals 2 and 3 shown in Figure 4 depolarized in response to one odor and hyperpolarized in response to another. Also, GGNs in different animals could respond differently to the same odor (Figure 4, hexanal). Almost a quarter of the odor-GGN pairs in our in vivo recordings showed reliable hyperpolarizations at some point in the odor response (40 out of 169). Earlier computational models(Papadopoulou et al., 2011; Kee et al., 2015) did not attempt to reproduce the variety of GGN responses we observed in vivo, including hyperpolarizations. To better understand the mechanisms underlying GGN's odor-elicited responses (and by extension, novel features of olfactory circuitry), we used our GGN model as the center of a more extensive mushroom body olfactory network.

\section{Sustained GGN responses require heterogeneous input to KCs}

We sought to determine olfactory network characteristics required to generate realistic responses in GGN because such an analysis could reveal gaps in our understanding of network function. We first tested whether simply simulating the average statistics of olfactory input to the mushroom body network would elicit responses from GGN like those observed in vivo. To test this, we extended our detailed GGN model with a full population of 50,000 simulated KCs. KCs are very small, have few dendritic branches, and generate action 
potentials; thus, in contrast to the large, complex, and passive GGN, the morphologies of

263 individual KCs are unlikely to differentially influence their odor coding properties. Therefore,

264 we used a relatively simple NEURON version of a single compartmental KC

model(Wüstenberg et al., 2004). Each model KC was connected to GGN in the $\alpha$ lobe via an

excitatory synapse, and each received inhibitory input from a random segment of GGN in the

calyx via a graded synapse (Figure 5a). To provide excitatory input to the $\mathrm{KCs}$, the firing

patterns of 830 PNs were simulated as spike-trains, and each KC was stimulated by $50 \%$ of

these spike trains via excitatory synapses(Jortner et al., 2007). The spike trains were designed to follow the statistics of PNs recorded in vivo(Mazor \& Laurent, 2005; Jortner et al., 2007). Thus, $77 \%$ of the PN spike trains were assigned a spontaneous firing rate of 2.6 spikes/s; during odor stimulation, $20 \%$ of these PNs were switched to 20 spikes/s modulated by the $20 \mathrm{~Hz}$ oscillations generated in the antennal lobe and reflected in the local field potential (LFP), and 10\% were inhibited (no spikes) (Figure 5b). In this model all the synapses between two given cell types had a fixed conductance. Simulations of this model produced a few highly synchronized bouts of spiking in the KC population (Figure $5 \mathrm{c}$ ), and corresponding isolated peaks in GGN's membrane potential (Figure 5d). These unrealistic responses were similar to those generated by earlier models(Papadopoulou et al., 2011; Kee et al., 2015).

Since this simplified model did not reproduce the long-lasting depolarization of GGN we had observed in vivo, we introduced more biological detail. It has been shown that synaptic strengths follow a lognormal distribution(Song et al., 2005; Loewenstein et al., 2011; Buzsáki \& Mizuseki, 2014). After we adjusted our network model to include this property, some KCs became weakly inhibited, allowing them to fire more volubly. We also adjusted the input to KCs provided by PNs. In our simplified model, input to the KCs emulated a fixed set of PNs all active throughout the duration of the odor stimulus. However, in vivo, spiking patterns of

287 PNs evolve over the course of an odor presentation, and different PNs respond to the same odor in different ways(Laurent \& Davidowitz, 1994; Stopfer et al., 2003; Mazor \& Laurent, 
2005), thus activating changing sets of KCs. To simulate these diverse responses, we divided the model's PN population into five groups: four groups responsive to the stimulus and one unresponsive. Odor-elicited spiking within each of the responsive groups started in a subset of its member PNs, and then, in each successive LFP cycle, new PNs were activated (Figure 5e). Lacking the heterogeneity in synaptic strengths onto KCs described above, even this complex activity pattern in PN population produced unrealistically synchronized bouts of activity in KCs, resulting in unrealistic isolated peaks in GGN's simulated membrane potential (Figure 5f, g). Increasing the strength of input from PNs allowed KCs to spike throughout the stimulus duration and generate sustained depolarization of GGN, but, under this condition, the number of spiking KCs became much higher than observed in vivo(Perez-Orive et al., 2002). Increasing the diversity of synaptic delays from PNs to KCs did not alleviate overly-synchronized spiking in the KC population if heterogeneity in synaptic strengths and temporal patterns of PN activity were omitted from the model (Figure 5 - figure supplement 1). However, we found that combining heterogeneous connectivity with structured PN firing patterns gave rise to GGN voltage traces that included sustained depolarization and temporal dynamics more characteristic of responses we had observed in vivo (Figure 5h, i). Moreover, even the steady PN activity shown in Figure 5b could produce sustained depolarization in GGN when the synaptic strengths were diversified (Figure 5 - figure supplement 2). This analysis, centered on GGN's responses, revealed important constraints for modeling the olfactory system.

\section{Some KCs fire at high rates in a GGN-centered olfactory network model}

310 The distribution of firing rates of the KC population in our simulations showed that, while

311 most KCs produced 0-2 odor-elicited spikes, a few KCs spiked much more (Figure 6a and

312 Figure 6 - figure supplement 1). To compare the firing rate distribution of KCs in the model

313 to that in vivo, we made patch clamp recordings from $147 \mathrm{KCs}$ in 114 animals, obtaining 314 results from $707 \mathrm{KC}$-odor pairs. On average, the spontaneous firing rates of these real KCs were very low $(\sim 0.09 \mathrm{~Hz})$ and reached only somewhat higher rates during odor presentation 
and after odor termination ( $\sim 0.15$ and $\sim 0.16 \mathrm{~Hz}$, respectively, Figure $6 \mathrm{~b})$, as previously

317 observed(Perez-Orive et al., 2002; Gupta \& Stopfer, 2014). Some odor-elicited responses in

$318 \mathrm{KCs}$, though, consisted of many more spikes. Figure 6c shows a representative example of

319 a hyperactive $\mathrm{KC}$, in which a 1s odor pulse elicited an average of 7 spikes (Figures $6 \mathrm{~d}$ and

320 e). Figure $6 f$ shows spike times obtained from 10 other KC-odor pairs. Overall, the distribution of spike counts in KCs we tested in vivo was clustered close to 0 but included a long rightward tail (Figure 6g), in agreement with our model (Figure 6a). KCs generating especially strong responses were not located in any particular part of the calyx, nor did they feature distinguishing morphologies (data not shown).

Although relatively rare $\mathrm{KC}$ odor responses with many spikes have been observed before (Perez-Orive et al., 2002; Gupta \& Stopfer, 2014), the origin and significance of these responses were unclear. We tested this with a series of simulations in which we systematically reduced the number of high spike-rate KCs by disconnecting them from the network. When KCs producing more than 5 odor-elicited spikes were removed (Figure 7 figure supplement 1), a different set of KCs emerged to replaced them by becoming more active; however, the replacement high spike-rate KCs were fewer in number than the original high-firing set (Figure 7a). As a result, the population spike time histogram and corresponding GGN voltage deflections became smaller (Figure $7 \mathrm{~b}$ and c). As we reiterated this process, deleting newly-emerged high spike-rate KCs from the network with each round, the rightward tail of the KC population's spike count distribution shortened, the number of low-spike KCs increased (Figure 7a), and, overall, the total number of spikes produced by the entire KC population decreased monotonically. Eventually, when no KCs in the reiterative simulation reached 5 odor-elicited spikes, we decreased the spike limit, first to 3 or more spikes, and then, to any spikes (Figure 7 - figure supplement 1 ). Throughout this

340 process, GGN's odor-elicited response gradually decreased, shifting from extended

341 depolarization to unrealistic, isolated peaks. Ultimately, no spiking $\mathrm{KCs}$, or responses in

342 GGN, remained (Figure 7c). Our analysis showed two things: first, the high spike-rate KCs in 
our model are not a static population, but are rather changing, emergent features of the

344 network's distribution of synaptic conductances, PN input, and GGN feedback; and second,

345 high-spike rate KCs augment the depolarization of GGN to realistic levels.

\section{Odor-evoked hyperpolarization in GGN originates in IG spiking}

347 Our intracellular recordings from GGN revealed extended periods of odor-elicited

348 hyperpolarization (Figure 4), something not previously reported in recordings made in vivo

349 and not explainable by existing models of GGN within its olfactory network. We hypothesized that these periods of hyperpolarization might originate in the activity of IG, a neuron known to share reciprocal inhibition with GGN (Figure 1a, right)(Papadopoulou et al., 2011).

352 Specifically, we hypothesized that an increase in IG activity might underlie the periods of 353 hyperpolarization in GGN, with IG's activity increase caused by disinhibition from GGN. To test this, we first tried adding a simple version of IG to our model following the reciprocal connectivity plan shown in Figure 1a. We tested a range of synaptic strengths and time constants for $\mathrm{GGN} \rightarrow \mid \mathrm{G}$, and IG $\rightarrow$ GGN synapses, and manipulated baseline firing rates of IG, but none of these efforts could generate odor-elicited hyperpolarization in GGN (data not shown). This hinted at a discrepancy in the causal connection between GGN and IG activity. Intuitively, if IG spiking increased solely because inhibition from GGN decreased, then IG spiking could only increase after GGN returned to baseline. However, as evident in Figure 8, sometimes GGN, recorded in vivo, hyperpolarized at the odor's onset, ruling out the possibility that disinhibition from GGN is the sole driver of spiking in IG.

The location and most properties of IG are unknown, and we were not able to identify it in our recordings. However, a previous report showed spikes in IG correlate one-to-one with IPSPs in GGN (Papadopoulou et al., 2011), suggesting we could infer IG's activity by examining GGN's membrane potential. Our recordings made in vivo from GGN revealed IPSPs in the absence of odors, and that odor presentations elicited an increase in the

368 frequency of IPSPs (Wilcoxon signed-rank test, $N=198$ pairs, $W=2328.5, p<<0.001$ ); 
several odors from 47 GGNs are shown as a peri-stimulus time histogram in Figures 8b and

372 (Papadopoulou et al., 2011), these results show that IG's responses to an odor pulse are

373 delayed and lengthy. Notably, as evident in Figure 8b, IG's firing rate begins to increase

374 before GGN's membrane potential returns to baseline, suggesting that IG's odor response cannot be driven solely by disinhibition from GGN. It has been hypothesized that IG receives input from KCs (Papadopoulou et al., 2011). Using our model, we could indeed reproduce realistic hyperpolarization in GGN's membrane potential by adding excitatory synapses to IG from either the PNs or the KCs (Figures 9a-e). Depending on the PN activity pattern, our simulations produced GGN membrane potentials with hyperpolarization and depolarization 380 (Figure 9f-i).

A remaining question concerned the source of excitation driving spontaneous activity in IG (Figure 9j, top black traces). KCs are nearly silent at rest(Perez-Orive et al., 2002; Gupta \& Stopfer, 2014), making it unlikely that they provide the sole source of excitation to IG. PNs, though, spike spontaneously because they receive direct, powerful input from spontaneously active ORNs(Joseph et al., 2012), suggesting a PN-driven pathway might be responsible for spontaneous activity in IG. To test this in vivo, we completely silenced PNs and KCs by bilaterally cutting the animal's antennal and labral nerves (Joseph et al., 2012) and then recorded intracellularly from GGN. We found that spontaneous IPSPs in GGN persisted in preparations with silenced PNs and KCs (Figure 9j, bottom red traces), demonstrating that KCs.

\section{Discussion}


olfactory coding. The unique giant GABAergic neuron GGN plays a central role in structuring olfactory codes in the locust mushroom body by regulating the excitability of KCs and parsing their responses into rhythmic bursts. We combined intracellular recordings from GGN and KCs, and developed a new morphologically detailed model of GGN as a focus of analysis to investigate GGN's properties, inputs, and outputs. Further, we used a broader model of the olfactory system built around GGN to explore several basic properties of the olfactory network. Our new electrophysiological recordings and computational model successfully reproduced the sparse activity of KCs and the membrane dynamics of GGN in the locust brain while providing concrete hypotheses about how the mushroom body circuit processes odor information.

It has been proposed that signals generated within GGN's $\alpha$ lobe branch propagate to its calyceal branch, where they provide global inhibition to all KCs(Papadopoulou et al., 2011).

408 It is clear that GGN inhibits KCs. But, the enormous size, extensive branching, and passive 409 conduction characterizing GGN raised the hypothesis that signals arising in GGN's $\alpha$ lobe 410 branch would attenuate to such an extent as they travel to the calyx that they would be 411 unable to effectively inhibit KCs. If this were the case, GGN would presumably inhibit KCs by 412 a different mechanism, such as through local inhibition entirely within the mushroom body calyx. Non-spiking interneurons in insects are often large with complex splays of neurites in separate brain areas, suggesting their far-flung branches may be functionally isolated, serving separate local computations(Burrows, 1981), and a variety of complex, local

416 processing has been proposed to occur within the locust mushroom body calyx (Leitch \&

417 Laurent, 1996). Our simulation of GGN's morphological and electrical properties shows that 418 realistic, odor-elicited levels of depolarizations of $10 \mathrm{mV}$ in GGN's $\alpha$ lobe branch do indeed 419 attenuate greatly with distance to amplitudes as low as $5 \mathrm{mV}$ in parts of the calyx. However, earlier studies of non-spiking neurons in invertebrates showed depolarizations of this amplitude should suffice to evoke neurotransmitter release. For example, (Burrows \& 
in the metathoracic ganglion of the locust can change the firing rate of its postsynaptic motor neuron. Similarly, (Manor et al., 1997) showed in a graded synapse in the lobster stomatogastric ganglion that voltage steps from $-50 \mathrm{mV}$ to $-45 \mathrm{mV}$ can reliably evoke postsynaptic effects. Thus, we conclude that GGN has the biophysical capacity to convey signals from KCs at GGN's $\alpha$ lobe branches to the calyx, providing effective global inhibition to all KCs.

GGN's arborizations in the calyx extend to different lengths, suggesting signals arising in the $\alpha$ lobe could attenuate more in some of its calyceal branches than in others. Our simulations indeed showed the amount of depolarization reaching GGN's distant branches varied with their locations, but only by a few millivolts (Figure $2 b$ ), within the range of variation caused by other factors. Consistent with this, APL, the Drosophila analog of GGN(Lin et al., 2014) shows different levels of $\mathrm{Ca}^{2+}$ activity in different spatial regions of its arbor when the animal is stimulated with low concentrations of odors (Inada et al., 2017). Inada et al. also found that some KCs are more effective than others at activating $A P L$, and that $A P L$, in turn, provides diverse levels of inhibition to different subsets of KCs in the same brain. By optogenetically activating a small subset of PNs, Inada et al. showed that APL could provide local inhibition to a subset of KCs, likely through dendrodendritic connections between KCs and APL within the calyx. Indeed, reconstructions of APL based on serial section electron microscope images show that it receives synaptic connections from KCs throughout its arbor, including within the calyx(Eichler et al., 2017; Zheng et al., 2018).

In the presence of strong odor stimuli, both GGN and APL appear to provide global inhibition

444 to all KCs(Papadopoulou et al., 2011; Lin et al., 2014), and, as we found (Figure 2), depolarizations evoked by strong input can remain above threshold to inhibit KCs throughout

446 GGN's arbor. We speculate that, in the locust, weaker stimuli might evoke lower levels of 447 depolarization in GGN's $\alpha$ lobe branch, leading to lower-amplitude signals that attenuate 448 below the threshold in GGN's distal branches needed to inhibit KCs. If, GGN, like APL, forms 449 reciprocal synapses with KCs within the calyx, it might be able to inhibit KCs there locally 
even in the absence of a global inhibitory signal. In this scenario, a KC driven to spike by a

weak odor stimulus would effectively inhibit only its close neighbors via local interactions

with GGN. Assuming randomly-connected input from PNs to KCs(Jortner et al., 2007; Caron

et al., 2013; Eichler et al., 2017), we can speculate that this circuitry could result in a winner-

take-all, center-surround-type of contrast enhancement in which only the most strongly

driven KCs in each region of GGN's arbor can respond to an odor. Given stronger odor

stimuli (or in the absence of local KC-GGN reciprocal connections in the calyx), signals originating in the $\alpha$ lobe would produce inhibitory output throughout GGN's arbor, dominate local inhibition, and allow KCs receiving inhibition from the same region of GGN to fire together, reducing the contrast among their responses.

Inhibition from GGN is known to sparsen the firing of KCs and to impose rhythmic time windows on their responses(Papadopoulou et al., 2011; Gupta \& Stopfer, 2012). Notably, our model also revealed that feedback inhibition from GGN can expand the dynamic range of inputs able to activate KCs, a result we confirmed in vivo with simultaneous recordings from GGN and KCs (Figure 3). This result is consistent with observations made in Drosophila where blocking inhibitory inputs to KCs narrowed the dynamic range of their odor responses(Inada et al., 2017). Why might expanding the dynamic range of KCs benefit olfactory coding? In the brain, a wide range of synaptic strengths exists even within a given type of neuron, and synaptic strength can change over time and with experience (reviewed in(Barbour et al., 2007)). As in other systems, homeostatic mechanisms might precisely and constantly tune conductances in KCs to match their inputs, enabling them to respond appropriately to a broad and changing range of inputs (reviewed in(Marder \& Goaillard, 2006)). Our results suggest an additional mechanism: feedback inhibition from GGN that helps KCs function robustly by expanding their sensitivities to a wider range of inputs, enabling them to generate consistent responses. 
477 2005), examples of KCs that fire substantially more spikes, spontaneously and in response

478 to odors, have been reported (Perez-Orive et al., 2002; Gupta \& Stopfer, 2014). Notably,

479 simulations that gave rise to realistic, sustained responses in GGN always included some

480 high-spike rate KCs. When we removed these high-firing rate KCs from the network, new

481 high-firing rate KCs arose to replace them (Figure 7). This analysis showed that high-firing

482 rate KCs are an emergent property of the olfactory network, determined by the balance of

483 input KCs receive: distributed patterns of excitation from PNs; and rhythmic inhibitory

484 feedback from GGN. Eliminating all high spike-rate KCs from a network had a modest effect

485 on GGN, slightly reducing the amplitude of its sustained, odor-elicited depolarization.

486 Eliminating KCs generating 3-6 spikes per stimulus reduced GGN's depolarization to

487 unrealistic levels, leaving only widely-separated peaks of activity (Figure 7).

488 Our intracellular recordings from GGN revealed more elaborate membrane potential

489 temporal dynamics than previously reported, including reliable and prolonged stimulus-

490 dependent periods of hyperpolarization, that varied with odor and animal. In our model,

491 simple reciprocal inhibition of GGN by the inhibitory neuron IG could not reproduce these

492 features. But we found introducing odor-elicited excitatory input to the inhibitory neuron IG

493 could give rise to hyperpolarizations of GGN's membrane potential (Figure 10). The direct

494 source of odor-elicited excitatory drive to IG is unknown, but could, in principle, be traced to

495 KCs. Indeed, a version of our model in which all KCs synapse upon IG reliably reproduced

496 fairly realistic odor-elicited hyperpolarizations in GGN. This result leads us to hypothesize

497 that KCs, which require strong and coincident input from PNs to spike (Perez-Orive et al.,

498 2002; Jortner et al., 2007) may serve as thresholding elements in the odor pathway from the

499 antennal lobe to IG, transferring odor-evoked activity, but not lower levels of spontaneous

500 activity, from PNs. The identity of this proposed odor pathway is not known.

501 Why are hyperpolarizations of GGN elicited by only some odors in some animals, as we saw

502 in vivo (Figure 4)? Our simulations suggest that odor-specific patterns of spiking across the

503 PN population (Stopfer et al., 2003) may underlie this variability (Figure 9b-i). Another 
504

505

506

507

508

509

510

511

512

513

514

515

516

517

518

519

520

521

522

523

524

525

526

527

528

possibility, not tested here, is that different sets of KCs could drive GGN and IG differentially such that odor-driven KCs strongly activate IG, but only weakly activate GGN, causing GGN to hyperpolarize. Different classes of KCs have been reported to differentially activate APL in Drosophila (Inada et al., 2017), but this possibility remains unexplored in locust.

Our results indirectly suggest IG is spontaneously active (Figures 8, 9). IG may generate this activity on its own or may inherit it from other olfactory or non-olfactory neurons. KCs are nearly silent at rest, making them unlikely direct sources of this activity, but it is possible that even extremely sparse spontaneous activity distributed over the population of 50,000 KCs could suffice to drive spontaneous spiking in IG. This possibility would be difficult to test in vivo.

In summary, we used biophysically detailed simulations in combination with electrophysiology performed in vivo to explore the olfactory circuit of the locust mushroom body. Our intracellular and patch recordings revealed new features of GGN, a neuron that plays a central role in shaping olfactory responses, and of the $\mathrm{KC}$ population. These results extend our understanding of the olfactory system, highlighting ways different components interact, and provide new constraints and predictions for additional research.

\section{Materials and Methods}

\section{Dissection and electrophysiology}

Newly eclosed adult locusts of both sexes picked randomly from our crowded colony were immobilized and the brain was exposed, desheathed and superfused with locust saline as described before(Brown et al., 2005). Our dissection included cutting the labral nerves and removing the suboesophageal ganglion (SOG), precluding stimulation via olfactory receptors in the palps. We usually obtained recordings from one GGN per animal and observed no systematic variation in results over the course of about a year. GGN is not visible from the 
529

530

531

532

533

534

535

536

537

538

539

540

541

542

543

544

545

546

547

548

549

550

551

552

553

554

555

brain's surface. To record from GGN, a sharp glass micropipette filled with 2 or 3M

potassium acetate with $5 \%$ neurobiotin was inserted into the peduncle region of the

mushroom body; when impaled, GGN could be identified by its characteristic pattern of

IPSPs in the voltage record (Figure 9j). At the end of the recording session, neurobiotin was

injected into the cell iontophoretically using $0.2 \mathrm{nA}$ current pulses at $3 \mathrm{~Hz}$ for 10 to 20

minutes, allowing the cell's identity to be confirmed by subsequent imaging. To analyze how the dynamic range of $\mathrm{KCs}$ is influenced by GGN, we calculated firing rate slopes, using the following method. After impaling GGN with a microelectrode, KCs were impaled with sharp microelectrodes filled with $1 \%$ neurobiotin in $0.5 \mathrm{M}$ potassium acetate. In $10 \mathrm{~s}$ trials we applied a $1 \mathrm{~s}$ current pulse to the $\mathrm{KC}$ at $2 \mathrm{~s}(A)$ and $7 \mathrm{~s}(B)$ and another current pulse to $\mathrm{GGN}$ only at $7 \mathrm{~s}$ (Figure 3e). Two amplitudes of current were tested ( $i_{1}$ and $\left.i_{2}\right)$. Currents injected into KCs were $0.02,0.05$ or $0.1 \mathrm{nA}$, and into GGN were $0.2,0.3,0.5,0.9$ or $1.0 \mathrm{nA}$, based on pretests to determine currents that effectively depolarized each cell. We counted the number of spikes in the KC over multiple trials and calculated the average firing rate $f$ during current injection under each condition. The slope of firing rate without GGN stimulation was computed as $m_{A}=\frac{f_{1, A}-f_{2, A}}{i_{1}-i_{2}}$, and the slope with GGN stimulation was $m_{B}=\frac{f_{1, B}-f_{2, B}}{i_{1}-i_{2}}$.

To test whether IPSPs in GGN originate from spontaneous activity in PNs, we first silenced the PNs by cutting both antennal nerves at the base(Joseph et al., 2012) and then searched for GGN, which could be identified by its ongoing unique pattern of IPSPS and confirmed by its morphology, revealed by subsequent filling with neurobiotin and imaging.

For patch clamp recordings from $\mathrm{KCs}$, the initial dissection was performed as described above. Patch pipettes were pulled to between 7 and $12 \mathrm{M} \Omega$, filled with locust internal solution(Laurent et al., 1993) as well as a neural tracer for subsequent histology (either 12 $\mathrm{mM}$ neurobiotin for later conjugation with an Avidin-Alexa complex, or $20 \mu \mathrm{M}$ Alexa Fluor tracer with absorption wavelengths of 488,568 or 633$)$. Patch recordings were made in 
556

557

558

559

560

\section{Stimulus delivery}

562

For GGN recordings, odor pulses were delivered to the ipsilateral antenna as described in

563

564

565

566

567

568

569

570

571

572

573

574

575

576

577

578

579

580 Imaging and neuronal tracing

581 Brains were dehydrated in an ethanol series and cleared with methyl salicylate or

582 CUBIC(Susaki et al., 2014), mounted in methyl salicylate or mineral oil respectively, and 
583

584

585

586

587

588

589

590

591

592

593

594

595

596

597

598

599

600

601

602

603

604

605

606

imaged with a Zeiss LSM 710 confocal microscope. Two GGNs were traced in detail from 3D image stacks using NeuroLucida software (MBF Bioscience, Williston, Vermont). The traces were converted to SWC format for further processing and cleanup using NLMorphologyConverter (www.neuronland.org). The two traces were very similar, and models based on both gave similar results. Here, we show results obtained from the GGN imaged at higher resolution.

\section{Statistics}

For each of 198 GGN-odor pairs, average IPSP rates were calculated over 5 trials in windows beginning $2 \mathrm{~s}$ before, and $2 \mathrm{~s}$ after, odor presentations. Wilcoxon-signed rank tests from the scipy package were used to compute the statistic and the two-sided $p$-value.

\section{Computational model}

GGN morphologies in SWC format were imported into NEURON and converted into passive models in NEURON's hoc format. The resting membrane potential was set to $-51 \mathrm{mV}$, as we have observed in vivo. The single compartmental KC model reported by (Wüstenberg et al., 2004) was translated manually into a NEURON model. This Hodgkin-Huxley type model included fast and slow $\mathrm{Na}+$ channels, and delayed rectifier, transient $\mathrm{A}$ type, and slow transient outward $\mathrm{K}+$ channels. The passive reversal potential of the $\mathrm{KCs}$ was set to $-70 \mathrm{mV}$. Custom Python scripts were written to set up network models and simulation experiments using NEURON's Python interface. The simulations were run on NIH's Biowulf supercomputer cluster (http://hpc.nih.gov) and simulation results were saved in HDF5 based NSDF format(Ray et al., 2015) and later analyzed with custom Python scripts. 

times the average spiking rate was further superimposed on odor-elicited spiking to model

613 oscillatory activity generated in the antennal lobe. Based on our own observations in vivo we

614 set $10 \%$ of spontaneously active PNs to respond with inhibition to odor presentations. We used a non-homogeneous Poisson generator to create the spike trains based on these rates.

To test the significance of varying temporal structure in PN firing patterns, we assumed

$620-30 \%$ of the PNs were unresponsive to odors, and divided the other $70 \%$ into 4 equally sized groups with the following odor-elicited sequences of excitation (E) and inhibition (I): with new PNs recruited during each LFP cycle(Mazor \& Laurent, 2005). Each group started its excitatory epoch with activation of $70 \%$ of its members and $10 \%$ were recruited in each of the next three LFP cycles. In this scheme at most $\sim 30 \%$ of PNs were active at any given time during odor presentations.

\section{Connectivity from GGN to KCs}

630 Each $\mathrm{KC}$ received one graded inhibitory synaptic input from a randomly assigned point on

631 GGN's calyceal branches. The strength of each synapse was adjusted to keep the KC's

632 membrane potential close to $-60 \mathrm{mV}$ when bombarded by spontaneous activity from PNs, as 633 observed in vivo(Joseph et al., 2012). The graded synapse was modeled as a NEURON 
634 mechanism based on published descriptions(Manor et al., 1997; Papadopoulou et al., 2011).

635 In some simulations the individual synaptic conductances were selected from a lognormal

636 distribution with the mean adjusted to produce realistic KC activity, i.e., to evoke spiking in

637 about $10 \%$ of the KCs with most of them responding with fewer than 5 spikes to odor stimuli.

638 The standard deviation of the lognormal distribution had the same value as the mean, based

639 roughly on (Song et al., 2005). In network simulations we considered KC activity to be

640 realistic if $\sim 10 \%$ or fewer KCs spiked in response to odor stimulus. Also, we considered KC

641 activity to be unrealistically low if it failed to depolarize GGN.

642

\section{Connectivity from PNs to KCs}

644 Half of the PN population was randomly and independently selected as presynaptic partners

645 for each KC. If two subsets of size $m$ and $n$ are randomly and independently selected from a 646 set of size $q$, the expected size of their intersection is $s=m * n / q$. Thus, with 800 PNs and 647 each KC receiving input from 400 PNs, the expected number of PNs shared by any two KCs 648 is $400 * 400 / 800=200$, i.e., they share about $50 \%$ of their presynaptic PNs, as shown in 649 vivo(Jortner et al., 2007). We carried out a parameter search to obtain a preliminary estimate 650 of a $\mathrm{PN} \rightarrow \mathrm{KC}$ synaptic conductance sufficient to evoke a few spikes in a single $\mathrm{KC}$ model 651 when driven with the expected combined PN firing rate during odor stimuli. Later, in the full 652 network model, we tested values around this conductance along with different GGN $\rightarrow \mathrm{KC}$ conductances. For heterogeneous conductance models, the conductances of individual synapses were selected from a lognormal distribution with the mean adjusted to produce realistic KC activity, as described above.

656

657 To test whether diverse synaptic delays could alleviate synchronized spiking in KC

658 population, we created a set of networks where $\mathrm{PN} \rightarrow \mathrm{KC}$ synapses were assigned a range of 659 delays from a normal distribution with mean $7.5 \mathrm{~ms}$ and standard deviation $3 \mathrm{~ms}$. We chose 660 these values with the animal's physiology in mind, by assuming a conservative speed of 0.2 
$661 \mathrm{~m} / \mathrm{s}=200 \mu \mathrm{m} / \mathrm{ms}$ for signal propagation through dendrites(Larkum et al., 1996). The length

662 of PN axon is about $1500 \mu \mathrm{m}$, so the total time delay from PN to KCs should be under 1500 /

663 $200=7.5 \mathrm{~ms}$, consistent with the delay of $6 \mathrm{~ms}$ observed in vivo by (Jortner et al., 2007).

664 The maximum diameter of a large $\mathrm{KC}$ dendritic arbor is about $600 \mu \mathrm{m}$ in the calyx and thus, the longest delay between two KCs receiving spikes from the same PN in their soma should be about $3 \mathrm{~ms}$.

667

\section{IG model and connectivity}

To simulate IG, we used a single compartmental Izhikevich-type model of a regular spiking

670 (RS) pyramidal cell from the Model DB repository

671 [https://github.com/ModelDBRepository/39948] modified to include graded synaptic input from GGN. To model IG's spontaneous firing, sufficient current was injected into it to

673 generate $\sim 7$ spikes/s. A single inhibitory synapse with $-80 \mathrm{mV}$ reversal potential connected

674 IG to one of GGN's basal dendrite segments. The strength and time constant for this

675 synapse were adjusted to produce IPSP amplitudes matching those we observed in vivo.

676 The same GGN segment was connected to IG via a graded synapse. In some simulations

677 either the output of all PNs or the output of all KCs were connected to IG via excitatory synapses. The synaptic weights from KCs to IG were selected from a lognormal distribution.

679

680

Data Analysis

681 Most analyses and 3D visualizations were carried out with custom Python scripts using

682 published modules including numpy, scipy, networkx, matplotlib, h5py, pandas and

683 scikitslearn.

684 Analyses of patch clamp recordings from KCs were carried out with custom MATLAB scripts. 685 
687 The morphological reconstruction of GGN will be made publicly available in neuromorpho repository (http://neuromorpho.org/).

689 The code for setting up and simulating the model is available on request and will be made 690 publicly available in ModelDB repository (https://senselab.med.yale.edu/ModelDB/). Scripts for data analysis are available at github: https://github.com/subhacom/mbnet_analysis.git.

692

693 Acknowledgements: We thank Tianming Li and lan McBain for help with tracing GGN morphology from confocal image stacks, Nitin Gupta (IIT Kanpur, India) for providing a confocal image stack of GGN for a pilot model and helpful advice for making recordings from GGN. We thank the members of the Stopfer lab for feedback and suggestions and Diantao Sun and Kui Sun for excellent animal care. We also thank Vincent Schram and Lynn Holzclaw of the NICHD Microscopy and Imaging Core for help with confocal imaging, George Dold and Bruce Pritchard of NIMH Section on Instrumentation for help with electrophysiology data acquisition setup, and Theodor Usdin, $\mathrm{NIMH} / \mathrm{NIH}$, for suggestions on tissue clearing and for providing initial reagents for the CUBIC protocol. We thank the Developmental Studies Hybridoma Bank for the nc82 antibody. This work utilized the computational resources of the NIH HPC Biowulf cluster (http://hpc.nih.gov). This work was funded by an intramural grant from NIH-NICHD to M.S.

Author contributions: S.R. and M.S. designed the study. Z.A. carried out KC electrophysiology and analyzed data. S.R. carried out GGN and KC electrophysiology, developed computational models and analyzed data. S.R. and M.S. wrote the manuscript. 


\section{References}

712 Arena, P., Calí, M., Patané, L., Portera, A. \& Strauss, R. (2015) Modelling the insect

713 Mushroom Bodies: Application to sequence learning. Neural Networks, 67, 37-53.

714

715 Barbour, B., Brunel, N., Hakim, V. \& Nadal, J.-P. (2007) What can we learn from synaptic weight distributions? Trends in Neurosciences, 30, 622-629.

717

718 Bathellier, B., Buhl, D.L., Accolla, R. \& Carleton, A. (2008) Dynamic Ensemble Odor Coding in the Mammalian Olfactory Bulb: Sensory Information at Different Timescales. Neuron, 57, 586-598.

721

722

Bazhenov, M., Stopfer, M., Rabinovich, M., Abarbanel, H.D.I., Sejnowski, T.J. \& Laurent, G.

723 (2001) Model of Cellular and Network Mechanisms for Odor-Evoked Temporal Patterning in the Locust Antennal Lobe. Neuron, 30, 569-581.

725

726

Brown, S.L., Joseph, J. \& Stopfer, M. (2005) Encoding a temporally structured stimulus with a temporally structured neural representation. Nature Neuroscience, 8, 1568-1576.

728

Burrows, M. (1981) Local interneurones in insects. In Roberts, A., Bush, B.M.H. (eds) Neurons without impulses:. Cambridge University Press, pp. 199-221.

Burrows, M. \& Siegler, M.V. (1978) Graded synaptic transmission between local interneurones and motor neurones in the metathoracic ganglion of the locust. $J$ Physiol, 285, 231-255. 
736 Buzsáki, G. \& Mizuseki, K. (2014) The log-dynamic brain: how skewed distributions affect network operations. Nat Rev Neurosci, 15, 264-278.

Cang, J. \& Isaacson, J.S. (2003) In Vivo Whole-Cell Recording of Odor-Evoked Synaptic

Carnevale, N.T. \& Hines, M.L. (2006) The NEURON Book.

743

Caron, S.J., Ruta, V., Abbott, L.F. \& Axel, R. (2013) Random convergence of olfactory inputs in the Drosophila mushroom body. Nature, 497, 113-117.

Curtis, H.J. \& Cole, K.S. (1938) Transverse Electric Impedance of the Squid Giant Axon. The Journal of General Physiology, 21, 757-765.

Demmer, H. \& Kloppenburg, P. (2009) Intrinsic Membrane Properties and Inhibitory Synaptic Input of Kenyon Cells as Mechanisms for Sparse Coding? Journal of Neurophysiology, 102, 1538-1550.

Eichler, K., Li, F., Litwin-Kumar, A., Park, Y., Andrade, I., Schneider-Mizell, C.M., 182. 
Friedrich, R.W. \& Laurent, G. (2001) Dynamic Optimization of Odor Representations by Slow Temporal Patterning of Mitral Cell Activity. Science, 291, 889-894.

762

Gentet, L.J., Stuart, G.J. \& Clements, J.D. (2000) Direct measurement of specific membrane capacitance in neurons. Biophys J, 79, 314-320.

765

766

Gupta, N. \& Stopfer, M. (2012) Functional Analysis of a Higher Olfactory Center, the Lateral Horn. The Journal of Neuroscience, 32, 8138-8148.

768

769

770

Gupta, N. \& Stopfer, M. (2014) A Temporal Channel for Information in Sparse Sensory Coding. Current Biology, 24, 2247-2256.

771

772

Hodgkin, A.L. \& Huxley, A.F. (1952) A quantitative description of membrane current and its application to conduction and excitation in nerve. J Physiol, 117, 500-544.

774

775

Hodgkin, A.L. \& Rushton, W.A.H. (1946) The Electrical Constants of a Crustacean Nerve Fibre. Proceedings of the Royal Society of London. Series B, Biological Sciences, 133, 444-479.

778

779

Inada, K., Tsuchimoto, Y. \& Kazama, H. (2017) Origins of Cell-Type-Specific Olfactory Processing in the Drosophila Mushroom Body Circuit. Neuron, 95, 357-367.e354. Coding in an Olfactory System. The Journal of Neuroscience, 27, 1659-1669. 
Joseph, J., Dunn, F.A. \& Stopfer, M. (2012) Spontaneous Olfactory Receptor Neuron Activity Determines Follower Cell Response Properties. The Journal of Neuroscience, $\mathbf{3 2}$, 2900-2910.

Kay, L.M. \& Stopfer, M. (2006) Information processing in the olfactory systems of insects and vertebrates. Seminars in Cell \& Developmental Biology, 17, 433-442.

791

Kee, T., Sanda, P., Gupta, N., Stopfer, M. \& Bazhenov, M. (2015) Feed-Forward versus Feedback Inhibition in a Basic Olfactory Circuit. PLOS Comput Biol, 11, e1004531.

Large, A.M., Vogler, N.W., Canto-Bustos, M., Friason, F.K., Schick, P. \& Oswald, A.-M.M. (2018) Differential inhibition of pyramidal cells and inhibitory interneurons along the rostrocaudal axis of anterior piriform cortex. PNAS, 115, E8067-E8076.

798

Large, A.M., Vogler, N.W., Mielo, S. \& Oswald, A.-M.M. (2016) Balanced feedforward 800 801 inhibition and dominant recurrent inhibition in olfactory cortex. PNAS, 113, 2276-

802

Larkum, M.E., Rioult, M.G. \& Luscher, H.R. (1996) Propagation of action potentials in the 804 dendrites of neurons from rat spinal cord slice cultures. J Neurophysiol, 75, 154-170.

805

Laurent, G. (1991) Evidence for voltage-activated outward currents in the neuropilar membrane of locust nonspiking local interneurons. Journal of Neuroscience, 11, 1713-1726. 
810 Laurent, G. \& Davidowitz, H. (1994) Encoding of Olfactory Information with Oscillating Neural Assemblies. Science, 265, 1872-1875.

812

813 Laurent, G. \& Naraghi, M. (1994) Odorant-induced oscillations in the mushroom bodies of 814 the locust. J. Neurosci., 14, 2993-3004.

815

816 Laurent, G., Seymour-Laurent, K.J. \& Johnson, K. (1993) Dendritic excitability and a voltage817 gated calcium current in locust nonspiking local interneurons. Journal of Neurophysiology, 69, 1484-1498.

819

820 Laurent, G., Wehr, M. \& Davidowitz, H. (1996) Temporal Representations of Odors in an Olfactory Network. The Journal of Neuroscience, 16, 3837-3847.

822

823 Leitch, B. \& Laurent, G. (1996) GABAergic synapses in the antennal lobe and mushroom body of the locust olfactory system. J. Comp. Neurol., 372, 487-514.

825

Lin, A.C., Bygrave, A.M., de Calignon, A., Lee, T. \& Miesenböck, G. (2014) Sparse, decorrelated odor coding in the mushroom body enhances learned odor discrimination. Nature Neuroscience, 17, 559-568.

829

830 Loewenstein, Y., Kuras, A. \& Rumpel, S. (2011) Multiplicative Dynamics Underlie the 831 Emergence of the Log-Normal Distribution of Spine Sizes in the Neocortex In Vivo. The Journal of Neuroscience, 31, 9481-9488. 
836

837

838

839

840

841

842

843

844

845

846

847

848

849

850

851

852

853

854

855

856

857

858

Luna, V.M. \& Pettit, D.L. (2010) Asymmetric rostro-caudal inhibition in the primary olfactory cortex. Nature Neuroscience, 13, 533-535.

MacLeod, K. \& Laurent, G. (1996) Distinct Mechanisms for Synchronization and Temporal Patterning of Odor-Encoding Neural Assemblies. Science, 274, 976-979.

Manor, Y., Nadim, F., Abbott, L.F. \& Marder, E. (1997) Temporal Dynamics of Graded Synaptic Transmission in the Lobster Stomatogastric Ganglion. Journal of Neuroscience, 17, 5610-5621.

Marder, E. \& Goaillard, J.-M. (2006) Variability, compensation and homeostasis in neuron and network function. Nat Rev Neurosci, 7, 563-574.

Mazor, O. \& Laurent, G. (2005) Transient dynamics versus fixed points in odor representations by locust antennal lobe projection neurons. Neuron, 48, 661-673.

Palmer, M.J. \& Harvey, J. (2014) Honeybee Kenyon cells are regulated by a tonic GABA receptor conductance. Journal of Neurophysiology, 112, 2026-2035.

Papadopoulou, M., Cassenaer, S., Nowotny, T. \& Laurent, G. (2011) Normalization for Sparse Encoding of Odors by a Wide-Field Interneuron. Science, 332, 721-725.

Peng, F. \& Chittka, L. (2017) A Simple Computational Model of the Bee Mushroom Body Can Explain Seemingly Complex Forms of Olfactory Learning and Memory. Current Biology, 27, 224-230. 
860 Perez-Orive, J., Bazhenov, M. \& Laurent, G. (2004) Intrinsic and Circuit Properties Favor

861 Coincidence Detection for Decoding Oscillatory Input. Journal of Neuroscience, 24,

862 6037-6047.

863

864 Perez-Orive, J., Mazor, O., Turner, G.C., Cassenaer, S., Wilson, R.I. \& Laurent, G. (2002)

865 Oscillations and Sparsening of Odor Representations in the Mushroom Body.

866 Science, 297, 359-365.

867

868

Poo, C. \& Isaacson, J.S. (2009) Odor Representations in Olfactory Cortex: "Sparse" Coding,

869 Global Inhibition, and Oscillations. Neuron, 62, 850-861.

870

871 Pouille, F., Marin-Burgin, A., Adesnik, H., Atallah, B.V. \& Scanziani, M. (2009) Input normalization by global feedforward inhibition expands cortical dynamic range. Nature Neuroscience, 12, 1577-1585.

874

875

Rall, W. (1964) Theoretical significance of dendritic trees for neuronal input-output relations.

876 In Reiss, R.F. (ed) Neural Theory and Modeling. Stanford Univ. Press., Stanford, CA,

877 pp. 73-97.

878

879 Raman, B., Joseph, J., Tang, J. \& Stopfer, M. (2010) Temporally diverse firing patterns in 880 olfactory receptor neurons underlie spatiotemporal neural codes for odors. J

881 Neurosci, 30, 1994-2006.

882 
883

884

885

886

887

888

889

890

891

892

893

894

895

896

897

898

899

900

901

902

903

904

905

906

907

Ray, S., Chintaluri, C., Bhalla, U.S. \& Wójcik, D.K. (2015) NSDF: Neuroscience Simulation Data Format. Neuroinform, 14, 147-167.

Roth, A. \& Häusser, M. (2001) Compartmental models of rat cerebellar Purkinje cells based on simultaneous somatic and dendritic patch-clamp recordings. J Physiol, 535, 445472.

Shimizu, K. \& Stopfer, M. (2017) A Population of Projection Neurons that Inhibits the Lateral Horn but Excites the Antennal Lobe through Chemical Synapses in Drosophila. Front. Neural Circuits, 11.

Song, S., Sjöström, P.J., Reigl, M., Nelson, S. \& Chklovskii, D.B. (2005) Highly Nonrandom Features of Synaptic Connectivity in Local Cortical Circuits. PLoS Biol, 3, e68.

Stettler, D.D. \& Axel, R. (2009) Representations of Odor in the Piriform Cortex. Neuron, 63, 854-864.

Stopfer, M., Jayaraman, V. \& Laurent, G. (2003) Intensity versus identity coding in an olfactory system. Neuron, 39, 991-1004.

Stuart, G. \& Spruston, N. (1998) Determinants of Voltage Attenuation in Neocortical Pyramidal Neuron Dendrites. Journal of Neuroscience, 18, 3501-3510.

Susaki, Etsuo A., Tainaka, K., Perrin, D., Kishino, F., Tawara, T., Watanabe, Tomonobu M., Yokoyama, C., Onoe, H., Eguchi, M., Yamaguchi, S., Abe, T., Kiyonari, H., Shimizu, 
908

909

910

911

912

913

914

915

916

917

918

919

920

921

922

923

924

925 Figure legends

926

927

928

929

930

931

932 157, 726-739. 743.e722.

Y., Miyawaki, A., Yokota, H. \& Ueda, Hiroki R. (2014) Whole-Brain Imaging with Single-Cell Resolution Using Chemical Cocktails and Computational Analysis. Cell,

Wüstenberg, D.G., Boytcheva, M., Grünewald, B., Byrne, J.H., Menzel, R. \& Baxter, D.A. (2004) Current- and Voltage-Clamp Recordings and Computer Simulations of Kenyon Cells in the Honeybee. Journal of Neurophysiology, 92, 2589-2603.

Zheng, Z., Lauritzen, J.S., Perlman, E., Robinson, C.G., Nichols, M., Milkie, D., Torrens, O., Price, J., Fisher, C.B., Sharifi, N., Calle-Schuler, S.A., Kmecova, L., Ali, I.J., Karsh, B., Trautman, E.T., Bogovic, J.A., Hanslovsky, P., Jefferis, G.S.X.E., Kazhdan, M., Khairy, K., Saalfeld, S., Fetter, R.D. \& Bock, D.D. (2018) A Complete Electron Microscopy Volume of the Brain of Adult Drosophila melanogaster. Cell, 174, 730-

Figure 1 GGN is a very large neuron, one per hemisphere, spanning a large portion of the locust brain. (a) Left hemisphere: Dye filled GGN (green) in a locust brain (magenta, visualized with nc82 antibody) with an overlaid KC tracing (orange, filled in a different brain). Dorsal: towards the viewer; ventral: into the page). Right hemisphere: Circuit diagram of the locust olfactory system. Arrows show known synaptic connections, + excitatory, - inhibitory. Scale bar: $200 \mu \mathrm{m}$. (b) Very thin, feather-like neurites from GGN wrap around and penetrate the peduncle of the mushroom body; these neurites are not included in our reconstruction. 
(c) GGN's neurites in the calyx have many bouton-like protrusions (d) whereas GGN's $\alpha$

lobe branches are relatively smooth. (b-d) scalebars: $100 \mu \mathrm{m}$. (e) 3D reconstruction of the same neuron shown in panel a viewed from (i) ventral and (ii) posterior side of the brain. Major branches shown in different colors. LCA: lateral calyx, MCA: medial calyx, LH: lateral

937 horn, a: anterior, p: posterior, d: dorsal, v: ventral, l: lateral and m: medial.

Figure 2 Passive voltage spread through GGN. (a) Simulation schematic: GGN's $\alpha$ lobe described in panel a lead to different steady state depolarizations of the GGN model at different locations; color indicates voltage change from resting potential; axial resistivity depolarization in the $\alpha$ lobe and the calyceal dendrites of GGN for conditions described in panel $\mathbf{a}$; width of gray area indicates density, white line median, and whiskers data range. (d) Schematic of simulation of multiple, random synaptic inputs to GGN: 500 synapses were connected to GGN's $\alpha$ lobe branch. (e) Bottom: raster plot of incoming spike times at all 500 synapses. The spikes arrived at each synapse at random times at linearly decreasing rate, starting with 20/s down to 0 after 500 ms. Top: for conditions shown in panel d, membrane potentials at randomly sampled terminal neurites in different regions of GGN. (f) Violin plots of peak depolarizations in the $\alpha$ lobe dendrites and the calyceal dendrites in model described in panel $\mathbf{d}$. (g) Violin plots of distribution of depolarizations in the calyx for different values of axial resistivity (RA, bottom) and membrane resistivity (RM, right) for the voltage clamp simulation described in panel $\mathbf{a}$. Lines connect the median results for specified RMs (right).

Figure 3 Feedback inhibition from GGN extends the dynamic range of KCs. (a) Model schematic: A single KC sends 50,000 excitatory synapses with random delays of 0-60 ms into GGN's $\alpha$ lobe branch. The KC receives feedback inhibition via a graded synapse from 
shown). (b) The model KC was tested with current pulses of increasing amplitude in three configurations: Isolated KC (left); KC receiving only spontaneous inhibition from GGN

961 (middle), and $\mathrm{KC}$ receiving spontaneous and odor-elicited feedback inhibition (right). As the amplitude of the current pulse increases, the KC's spiking first increases, and then, as the membrane potential nears saturation, decreases. (c) Comparison of the number of spikes evoked by current steps in $\mathrm{KC}$ in the three configurations shown in b. (d) Slopes of the rising parts of the KC-only (left, cyan) and KC with GGN feedback (right, orange) curves in c, normalized by the KC-only slope. (e) Intracellular current injection protocol: a current pulse of low (1) or high (2) amplitude was injected into the KC at 2-3 s (A) and again at 7-8 s (B), now accompanied by a depolarizing current pulse into GGN. (f) Somata of KCs (marked by arrowheads) recorded simultaneously with GGN (neurites visible) in one animal. Scale bar: $100 \mu \mathrm{m}$. (g) Slope of KC spike rate during current stimulation with respect to stimulating current amplitude when GGN was at baseline (left) and depolarized (right). $5 \mathrm{KCs}$ were tested in 4 animals; in all cases, slopes decreased with GGN activity, indicating dynamic range extension.

Figure 4 In vivo, GGN's responses to odors vary with odor and animal. Examples from 3 animals and 2 odors (horizontal gray bars, 1 s) (black traces: average of 5 trials, gray: standard error of the mean, data low-pass filtered at $49 \mathrm{~Hz}$ ). Response features include depolarization or hyperpolarization upon stimulus onset and/or offset, and depolarization or

978 hyperpolarization evoked by different odors in the same GGN.

979 Figure 5 Tuning the olfactory network by reference to GGN's olfactory responses required 980 heterogeneous synaptic strengths onto KCs and structured patterns of activity in PNs. (a)

981 Schematic of mushroom body network model. Each of the 50,000 KCs receives input from

$98250 \%$ of the 830 PNs, which are modeled as spike trains. All KCs excite GGN in its $\alpha$ lobe

983 branch and receive inhibition from a random calyceal branch of GGN. (b-d) Model with

984 simplified, homogenous firing patterns in PNs and uniform synaptic strengths generates

985 unrealistic membrane potential in GGN. (b) Raster plot of model PN spike trains (67 shown); 
986

987

988

989

990

991

992

993

994

995

996

997

998

999

1000

1001

1002

1003

1004

1005

1006

1007

1008

1009

1010

1011

1012

dots in each row mark spike times in a PN. (c) Raster plot of spike trains evoked in the KCs (397 shown) when all of them receive identically strong inhibitory connections from GGN. (d) Unrealistic membrane potential in GGN features a few peaks corresponding to highly synchronized bouts of activity in KCs. Light gray bar: 1 s odor stimulation. (e-g) Model in which subpopulations of PNs have different temporal patterns of spiking during and after odor stimulation generates unrealistic membrane potential in GGN. (e) Rasters show different firing patterns in different PNs. (f) Simulation of model with PN activity pattern in panel e along with uniform synaptic strengths onto KCs generate synchronized spiking in KCs and (g) unrealistic membrane potential in GGN. Simulation of a model with both structured PN activity patterns in panel $\mathbf{e}$ and heterogeneous synaptic strengths gives rise to (h) temporally diffuse spiking in KC population and (i) sustained depolarization of GGN similar to that observed in vivo (e.g. Figure 4 animal 1). Dark gray bar: 1 s odor stimulation; light gray bar: $0.5 \mathrm{~s}$ "off response" period.

Figure 6 In vivo, some $\mathrm{KC}$ responses are hyperactive, as predicted by simulations. (a)

Distribution of KC firing rates in simulation producing realistic GGN depolarization. (b) KC firing rate averaged over $707 \mathrm{KC}$-odor pairs. Shaded region indicates standard error of the mean. Black horizontal bar: $1 \mathrm{~s}$ odor stimulation. (c) A hyperactive response $\mathrm{KC}$ filled with dye (mushroom body calyx and pedunculus outlined with dashed lines (scale bar: $50 \mu \mathrm{m}$ ) and (d) its recorded membrane potential in response to odor stimulus, and (e) its average firing rate elicited by this stimulus computed in a $100 \mathrm{~ms}$ window. (f) Sample KC spike trains. (g) Histogram of average firing rate of KCs across trials upon odor presentation measured in a $1.4 \mathrm{~s}$ window. The last bin in the rightward tail includes two different KCs.

Figure 7 High spike-rate KCs contribute to sustaining GGN's odor-elicited depolarization. (a) Histogram of spike counts in KCs for one template network at the first and the last run of each spike count limit. (b) Population spike time histogram of KCs (100 ms bins) for the same simulations. (c) GGN membrane potential. See text for the procedure. Legend is same for a-c. 
1013 Figure 8 IPSPs in GGN suggest IG spikes upon odor presentation and is responsible for

1014 hyperpolarizations in GGN. (a) In vivo recordings of GGN's membrane potential from two

1015 animals showing IPSPs (arrowheads) believed to originate as spikes in IG. Vertical scale

1016 bar: $10 \mathrm{mV}$. (b) Peristimulus time histogram (PSTH) shows IPSP peak times from 1257

1017 odor-trials across 47 GGNs, presumably reflecting spikes in IG. Because IG begins to spike

1018 before GGN's membrane potential returns to baseline, IG's odor response cannot be driven

1019 by disinhibition from GGN. Black horizontal bar: odor presentation in panels a and b, which

1020 share the time axis. (c) Same as panel b but showing full responses.

1021 Figure 9 An additional, unidentified olfactory pathway to IG is needed to explain odor-elicited hyperpolarization in GGN. (a) Schematic of model with IG receiving direct excitation from KCs and reciprocal inhibition from GGN. (b) Spike raster of PN activity producing GGN

1024 hyperpolarization. (c) Spike raster of KC population activity in the same simulation. (d)

1025 Simulated GGN membrane potential including odor-elicited hyperpolarization mimics responses observed in vivo (e.g. Figure 4, Animal 3, hexanal). (e) Corresponding simulated

1027 IG membrane potential. This simulation included a 200 ms synaptic delay from KCs to IG. (fi) Varying the temporal pattern of PN population activity can produce different response pattern in the same network as e. (f) Raster plot of PN activity with a different temporal pattern from that in e. (g) Spike raster showing concomitant KC activity. (h) Simulated GGN membrane potential including odor evoked de- and hyperpolarization (similar to Figure 4, Animal 1 hexanal). (i) corresponding simulated IG membrane potential. (j) Spontaneous activity in IG does not originate in the antennal lobe. Top two black traces show spontaneous IPSPs in GGN's membrane voltage from two animals with intact olfactory systems. The bottom two red traces are from the left and the right GGN in another animal in which the antennal lobes had been silenced by cutting both antennal nerves. Vertical scale bar $5 \mathrm{mV}$,

1037 horizontal scale bar $1 \mathrm{~s}$. 
1038

1039

1040

1041

1042

1043

1044

1045

1046

1047

1048

1049

1050

1051

1052

1053

1054

1055

1056

1057

1058

1059

1060

1061

1062

Figure 10 Updated olfactory connectivity model where odor responses in the KC population are gated by feedback inhibition from GGN and inhibition of GGN via odor evoked spiking in IG, which itself receives input from an unknown olfactory pathway.

\section{Supplemental Information}

Video S1. Related to Figure 1: 3D view of the confocal stack of the dye filled GGN in Figure 1a.

Video S2. Related to Figure 2: 3D view of depolarizations (color coded spheres) at various locations in GGN arbor in simulation described in Figure 2a.

Figure 2 - figure supplement 1: Signal propagation time throughout GGN. Figure shows the time of peak depolarization at the lateral calyx (LCA) and medial calyx (MCA) branches of GGN with respect to that at the base of the $\alpha$ lobe branch in the simulation described in Figure 2d, e. Darker line in the middle indicate median and whiskers data range. The voltages were smoothed by $100 \mathrm{~Hz}$ lowpass filter.

Figure 2 - figure supplement 2: KC spiking vs GGN voltage deflection in simulations where all KCs received identical PN input for different values of maximum synaptic conductances onto KCs. GGN segments were grouped by peak depolarization from the resting potential and the total number of spiking KCs postsynaptic to these segments (top) or the total number of spikes in the KCs postsynaptic to these segments (bottom) were normalized by the total number of KCs (both spiking and nonspiking) post synaptic to these segments.

Figure 2 - figure supplement 3: Correlations between number of spikes in a KC and various parameters influencing spiking: $\Delta \mathrm{V}_{\mathrm{m}}$ - peak voltage deflection of its presynaptic GGN segment, $\bar{g}_{G G N}$ - maximum conductance of the synapse from GGN, 
1063

1064

1065

1066

1067

1068

1069

1070

1071

1072

1073

1074

1075

1076

1077

1078

1079

1080

1081

1082

1083

1084

1085

1086

1087

1088

$\sum_{\text {presynaptic }}$ spikes $_{P N}-$ total number of spikes over all its presynaptic PNs, $\sum_{\text {presynaptic }} \bar{g}_{P N}-$ sum of maximum conductance of all PN synapses, $\sum_{\text {presynaptic }} \bar{g}_{P N} \times$ spikes $_{P N}-$ sum of the numbers of spikes in its presynaptic PNs weighted by the maximum conductances of their synapses onto this $\mathrm{KC}$. The negative correlation between the number of spikes in a $\mathrm{KC}$ and depolarization of its presynaptic GGN segment is very small in simulations of (a) models with temporally patterned PN response (as in Figure 5e) and synaptic strengths onto KCs selected from lognormal distributions, (b) models with steady activity in a fixed set of PNs (as in Figure 5b) and synaptic strengths onto KCs selected from lognormal distributions, and (c) models with steady activity in a fixed set of PNs and constant synaptic strengths onto KCs. Color indicates the number of KCs that spiked in the simulation.

Figure 3 - figure supplement 1: Model KC responses to current step injections while inhibited by pseudo-dynamic clamp of GGN voltage from a network model show inhibition expands dynamic range of $\mathrm{KCs}$. The test $\mathrm{KC}$ received no inhibition (left column), inhibition driven by weak GGN depolarization (middle column), or strong GGN depolarization (right column). The strong GGN voltage was obtained from the simulation of a full PN-KC-GGN network model. The weak GGN depolarization was derived by halving the deflections of the former from resting potential.

Figure 5 - figure supplement 1: Introducing diverse delays in PN to KC synapses does not alleviate the unrealistic extent of synchronization of $\mathrm{KC}$ firing. This model had architecture similar to that in Figure 5b-d, but here the synaptic delays from PNs to KCs were sampled from a normal distribution (mean=7.5 ms, standard deviation=3 ms).

Figure 5 - figure supplement 2: Model with steady activity in a fixed set of PNs (as in Figure 5b) can produce sustained depolarization of GGN when the synaptic strengths are lognormally distributed. Gray: 1 s odor stimulus.

Figure 6 - figure supplement 1: Example distributions of KC spike counts in four instantiations of the network architecture in Figure 6. 
1089 Figure 7 - figure supplement 1: Flow chart of the reiterative simulation of the same model

1090 while removing high-firing KCs. The KCs which spiked more than limit times in a simulation

1091 were eliminated from the model and this updated model was simulated again. The number of

1092 KCs producing more than limit spikes generally reduced after this, and as the process was

1093 repeated, at some point no KC met this criterion (i.e. no KC was removed from the model).

1094 Then we lowered the limit and repeated the process. This was continued until none of the

1095 KCs were able to spike. 
a

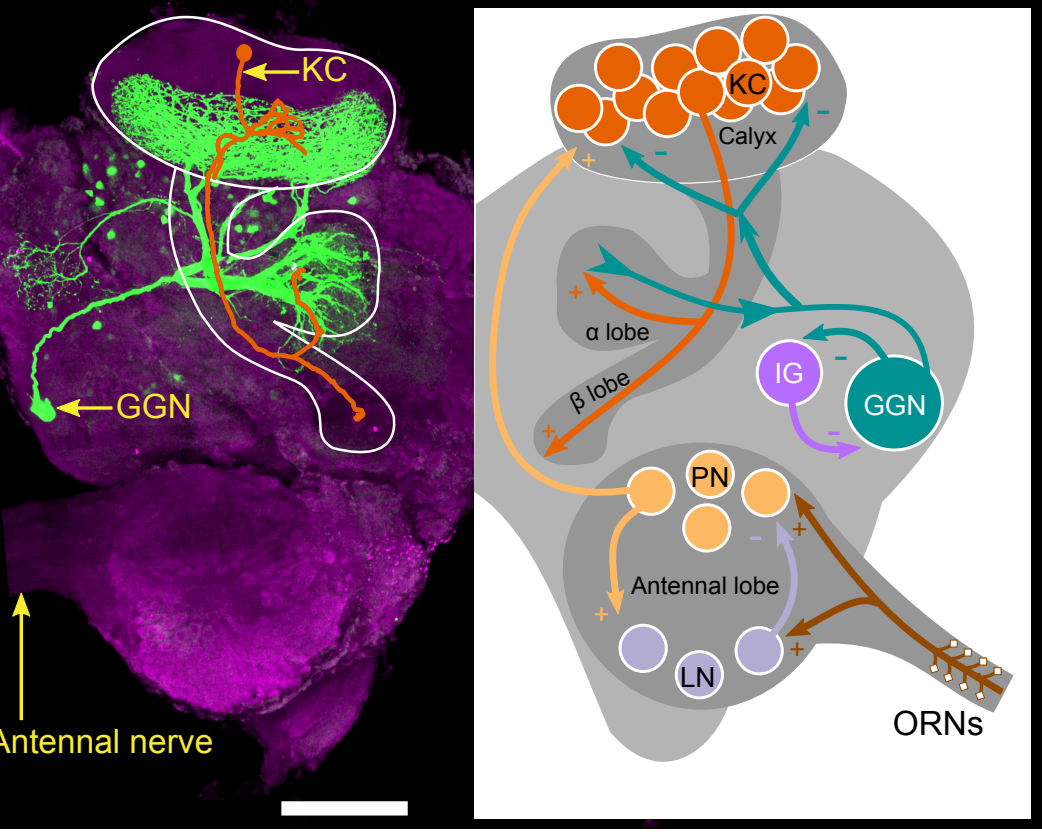

e

(i)

(ii)
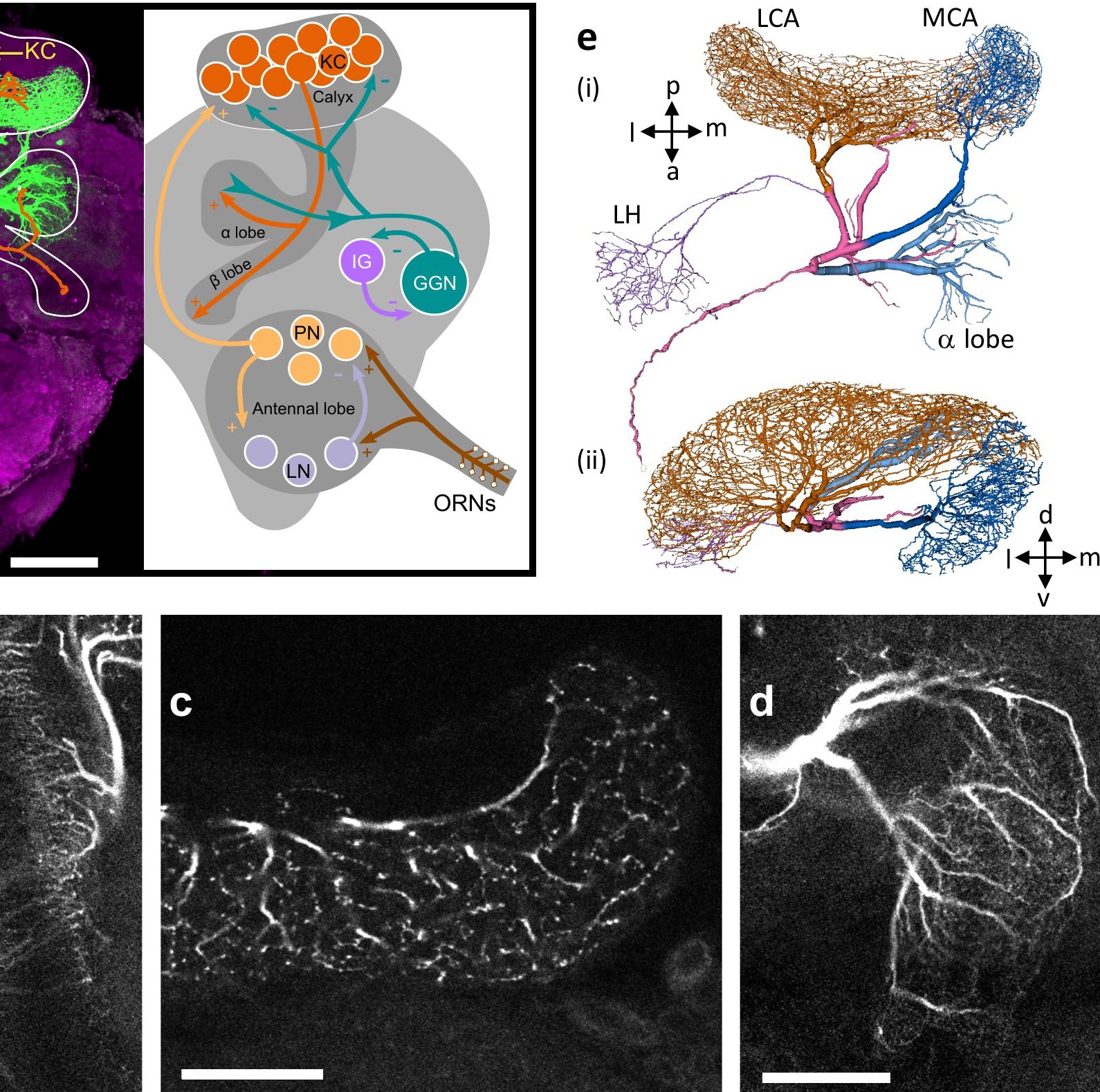

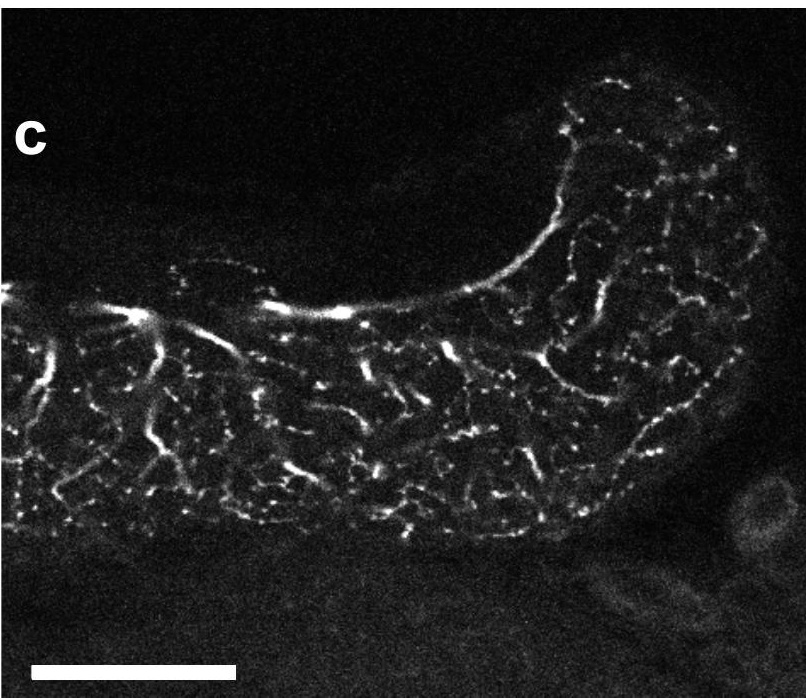

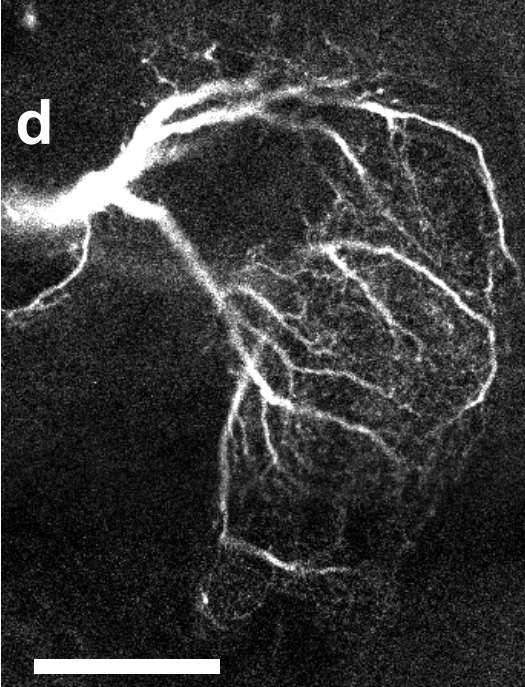

\section{b

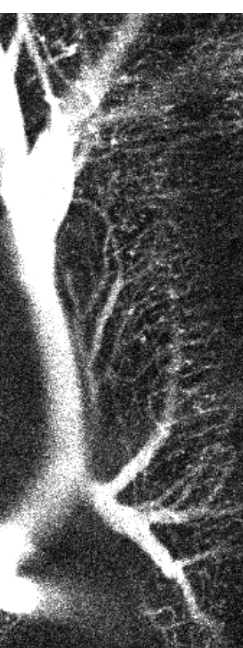


Figure 2 - figure supplement 1

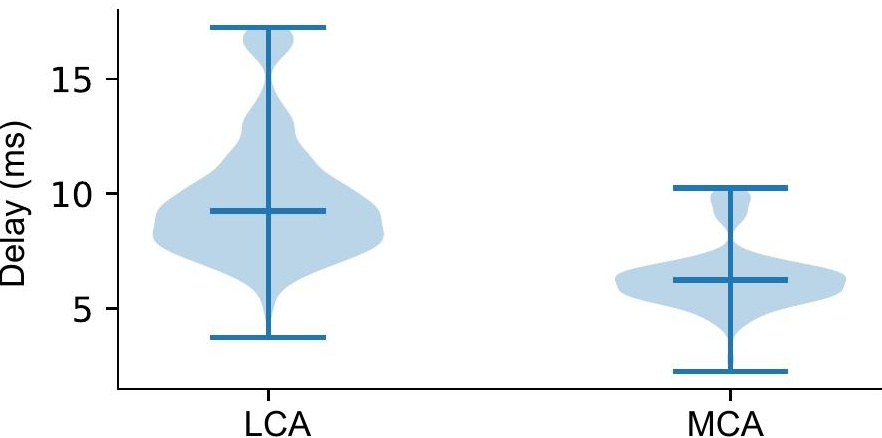




\section{Figure 2 - figure supplement 2}

$$
\begin{aligned}
\nabla \longrightarrow & \bar{g}_{\mathrm{PN} \rightarrow K C}=3.7 \mathrm{pS}, \overline{\mathrm{g}}_{\mathrm{GGN} \rightarrow \mathrm{KC}}=0.7 \mathrm{nS} \\
\hdashline & \overline{\mathrm{g}}_{\mathrm{PN} \rightarrow \mathrm{KC}}=3.7 \mathrm{pS}, \overline{\mathrm{g}}_{\mathrm{GGN} \rightarrow \mathrm{KC}}=0.5 \mathrm{nS} \\
\square & \bar{g}_{\mathrm{PN} \rightarrow \mathrm{KC}}=4.0 \mathrm{pS}, \overline{\mathrm{g}}_{\mathrm{GGN} \rightarrow \mathrm{KC}}=0.9 \mathrm{nS} \\
\longleftrightarrow & \overline{\mathrm{g}}_{\mathrm{PN} \rightarrow \mathrm{KC}}=4.5 \mathrm{pS}, \overline{\mathrm{g}}_{\mathrm{GGN} \rightarrow \mathrm{KC}}=0.9 \mathrm{nS}
\end{aligned}
$$
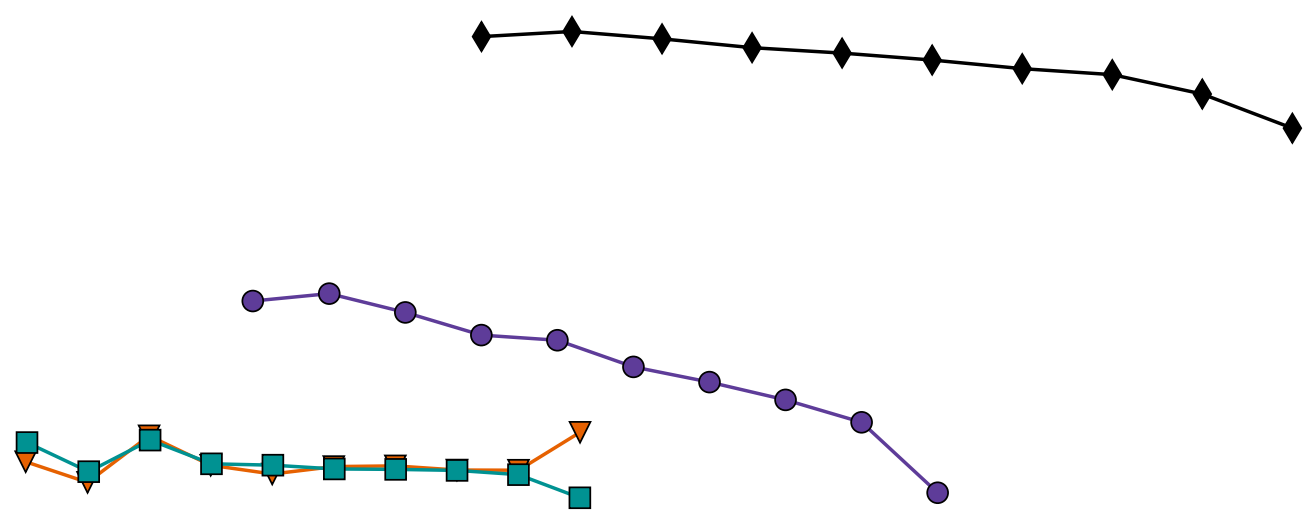

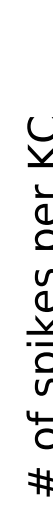

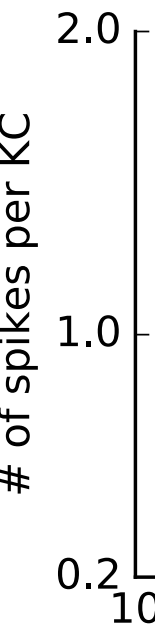
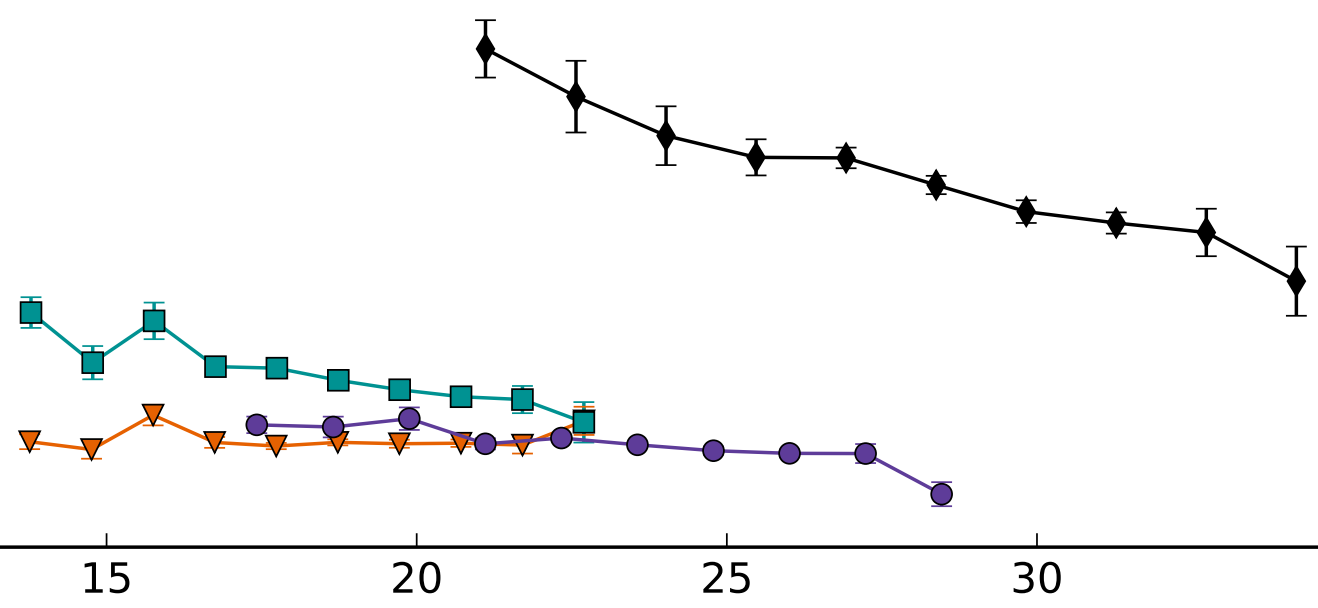

25

30 


\section{Figure 2 - figure supplement 3}

Number of spiking KCs

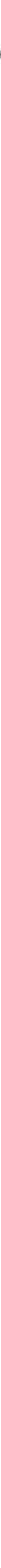




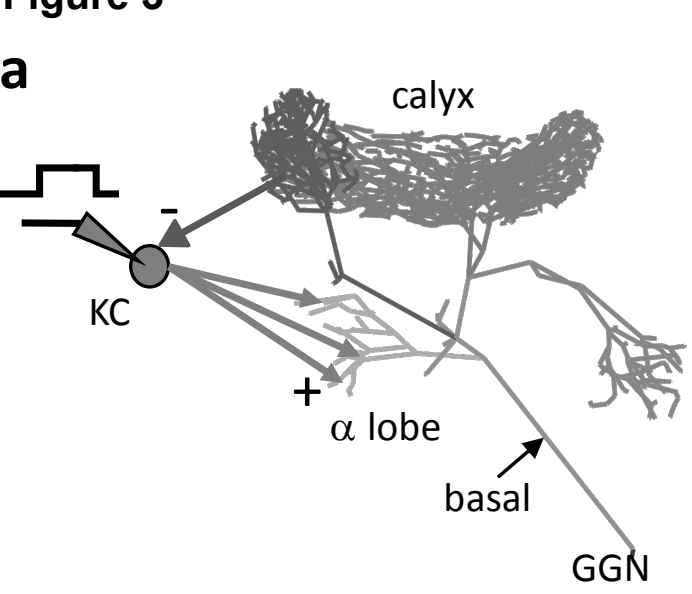

b

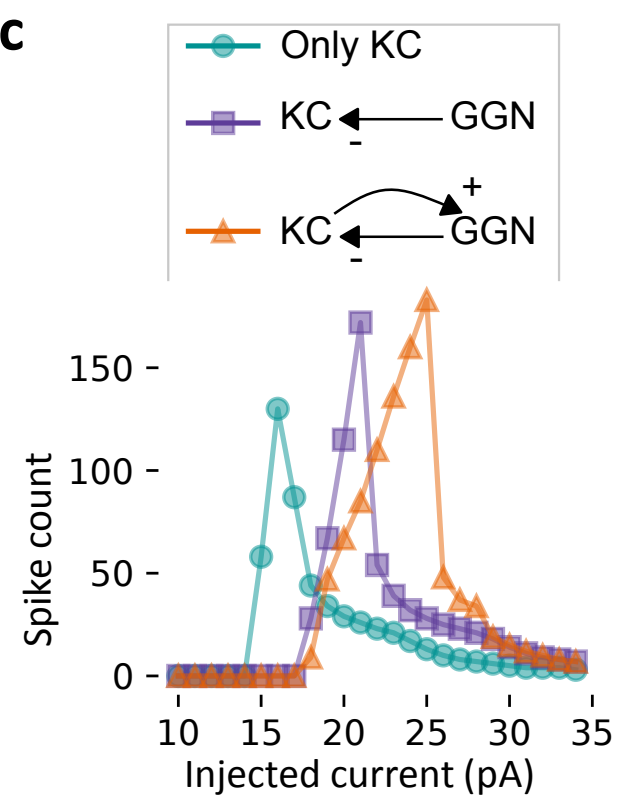

Only KC

$\mathrm{KC} \longleftarrow$ GGN

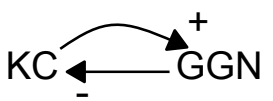

14

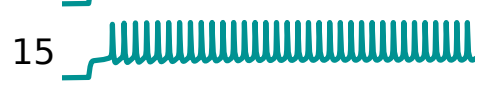

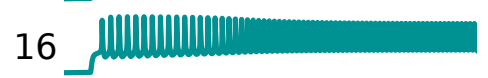

17

18

19

20

21

21
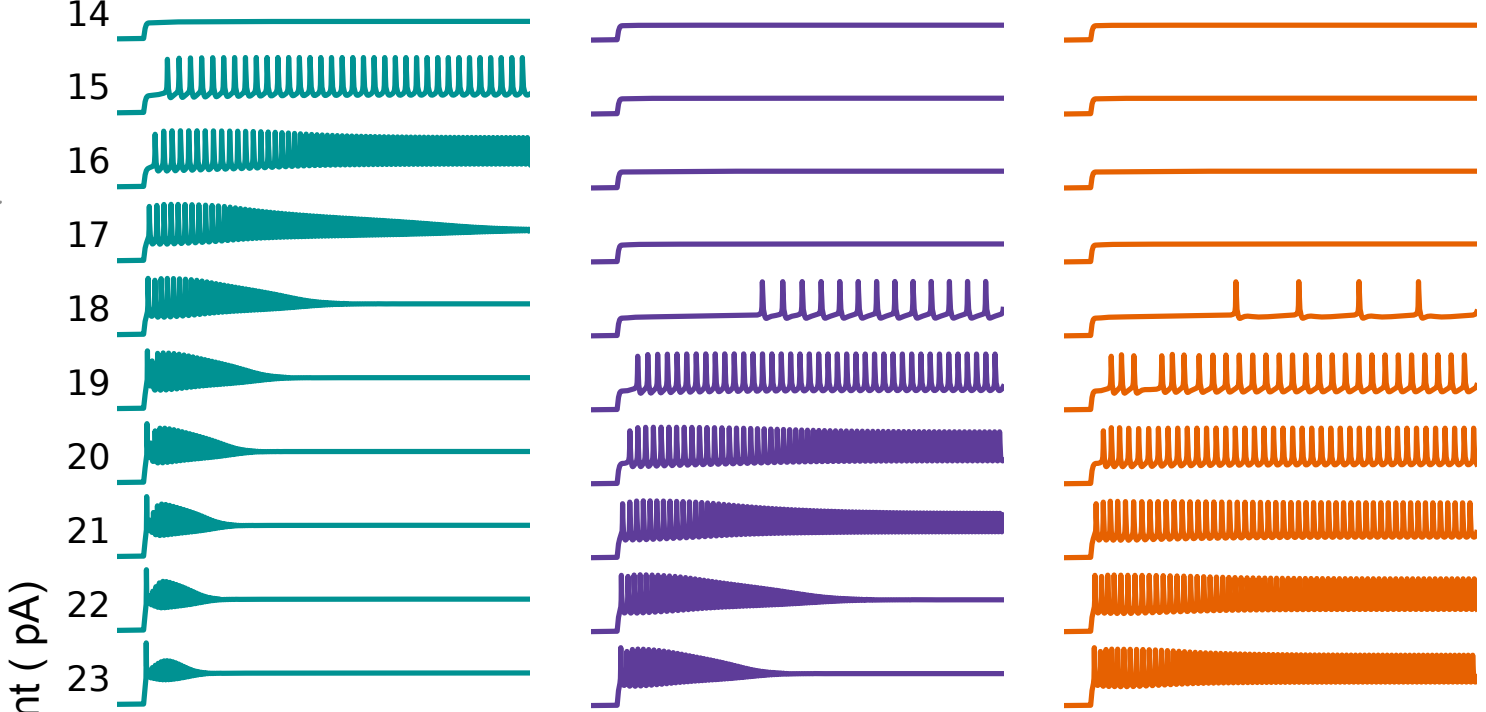

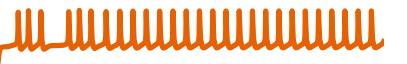
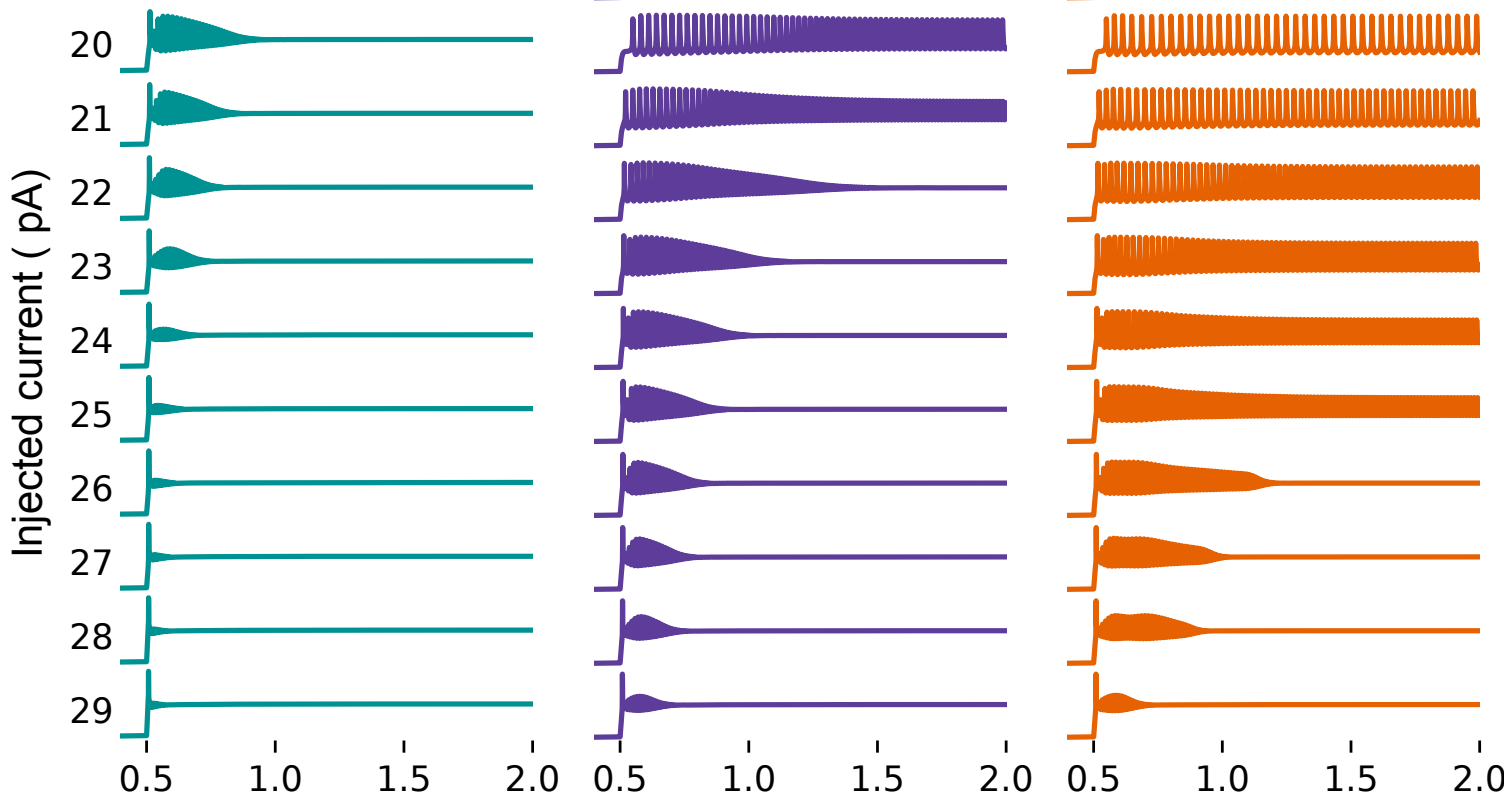
Time (s)
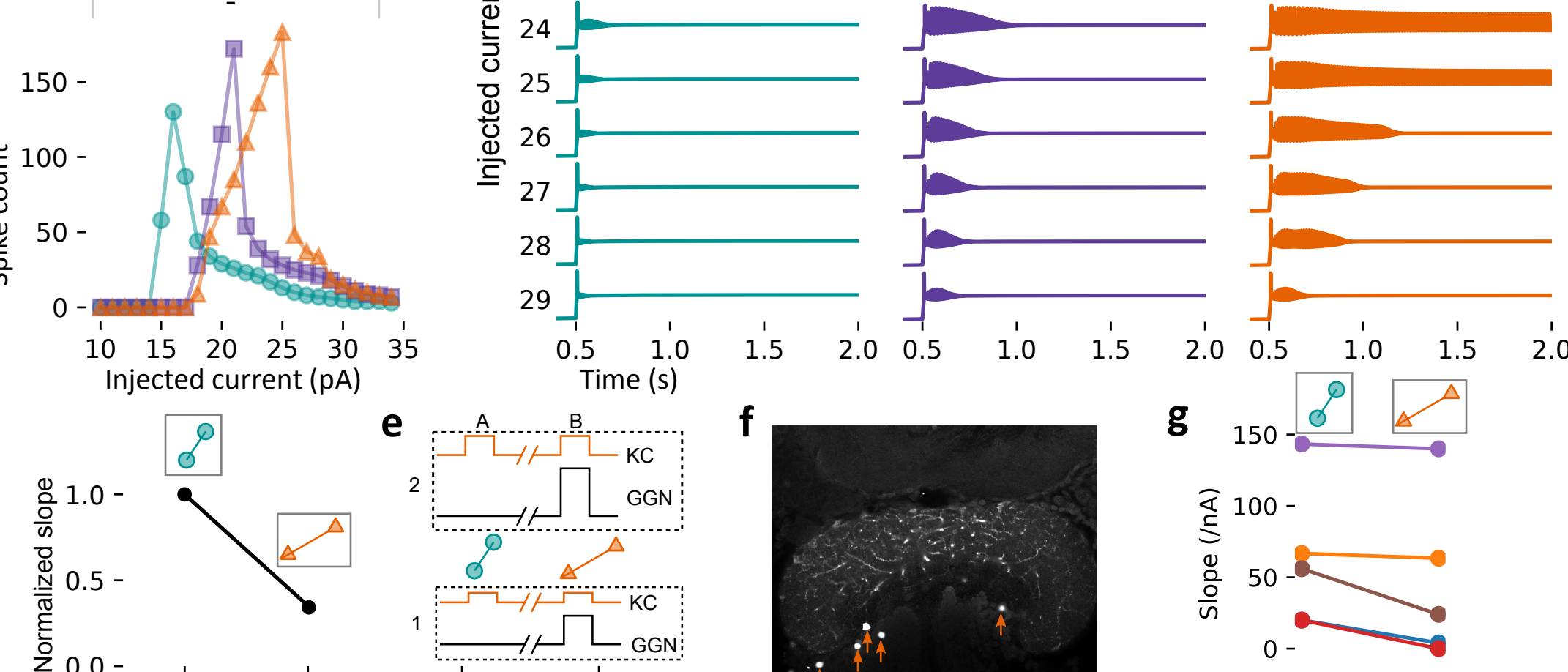

e

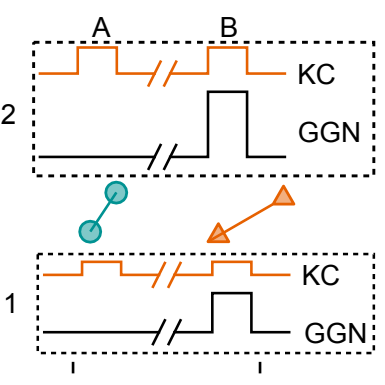

f

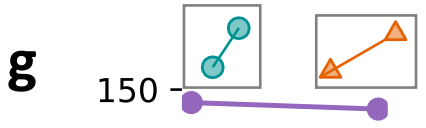

Only KC $\mathrm{KC}+\mathrm{GGN}$

Only KC

$\mathrm{KC}+\mathrm{GGN}$

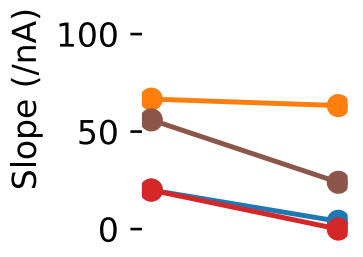

Only' KC KC+'GGN 
Figure 3 - figure supplement 1

Injected

Current

$\begin{array}{ll}\nwarrow & -50 \\ \Xi & -60 \\ \ddagger & -70\end{array}$

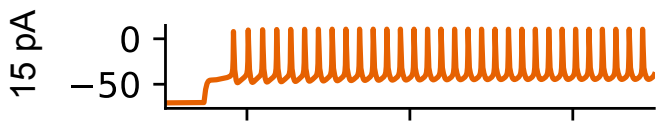

告

,

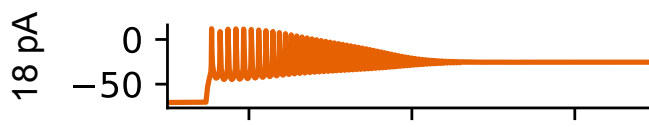

$\begin{array}{lr}\overleftarrow{a} & 0-1 \\ \stackrel{\square}{\circ} & -50-1\end{array}$

$\begin{array}{lr}\frac{1}{a} & 0 \\ \stackrel{N}{N} & -50\end{array}$

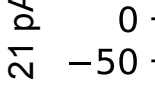

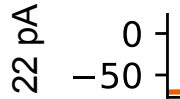

$\stackrel{\frac{\pi}{2}}{\mathrm{~N}}-50-$

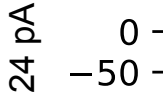

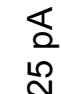

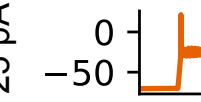

$\underset{\substack{a \\ 0}}{0}$

$\begin{array}{rr}0 & 0 \\ 0 & -50\end{array}$

N -50

$<$

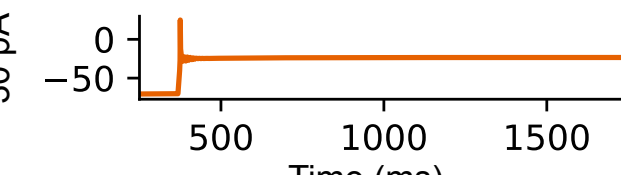

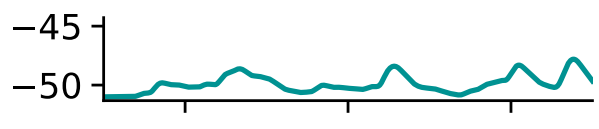
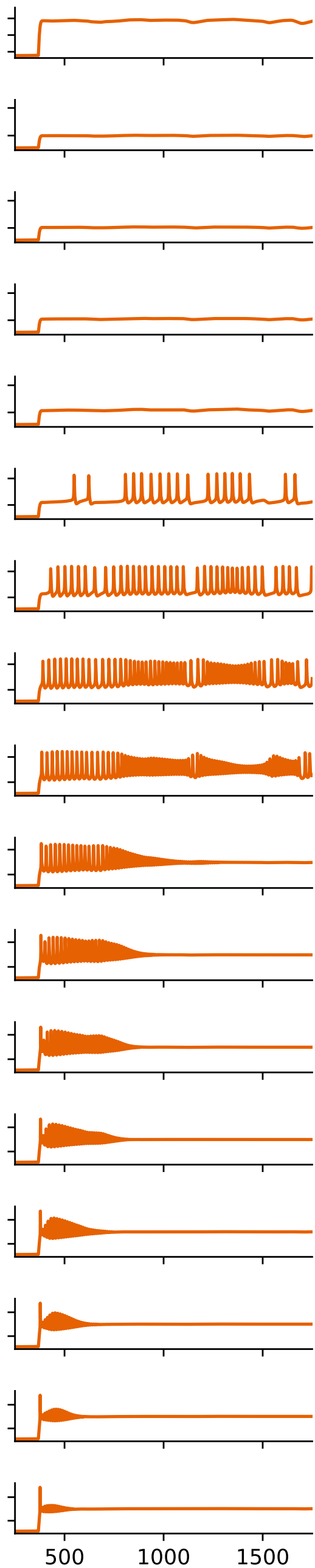
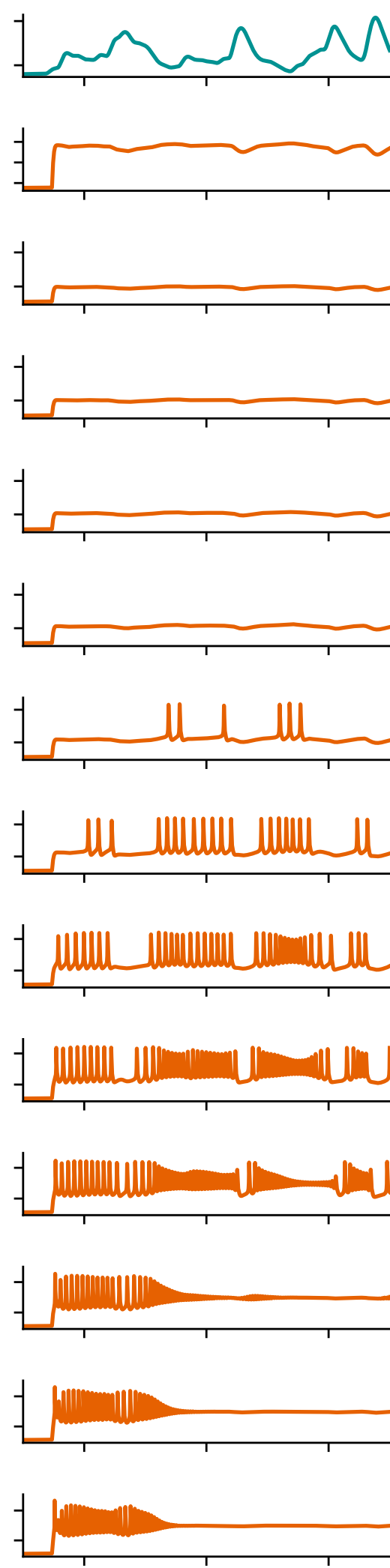

$-1$

$-1$

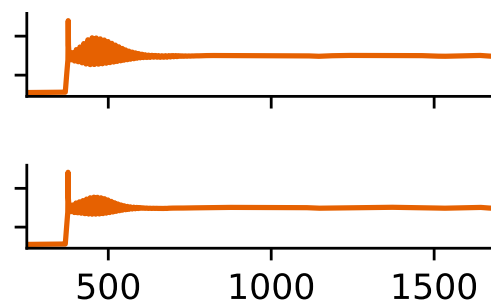




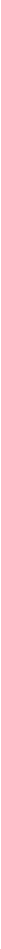


a PNs conporitopososo

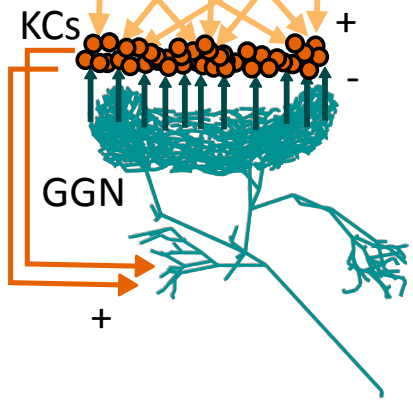

z

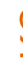

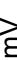

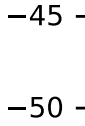

C

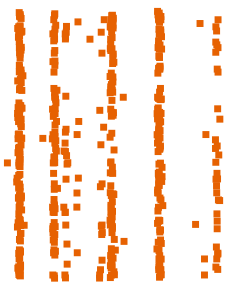

e

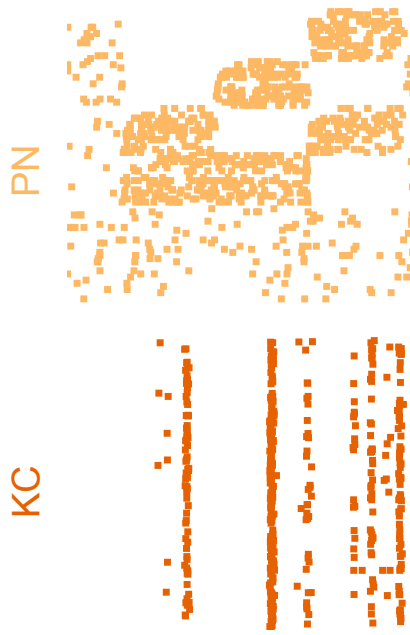

g $-45-$
ह $-50-$

GGN

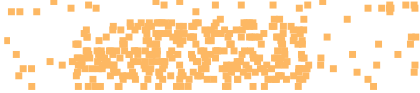

h

$\underline{Y}$

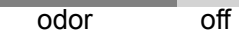

"

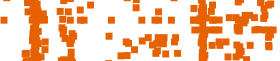

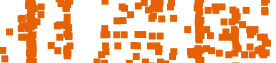

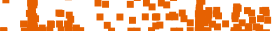

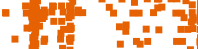

늘 
Figure 5 - figure supplement 1

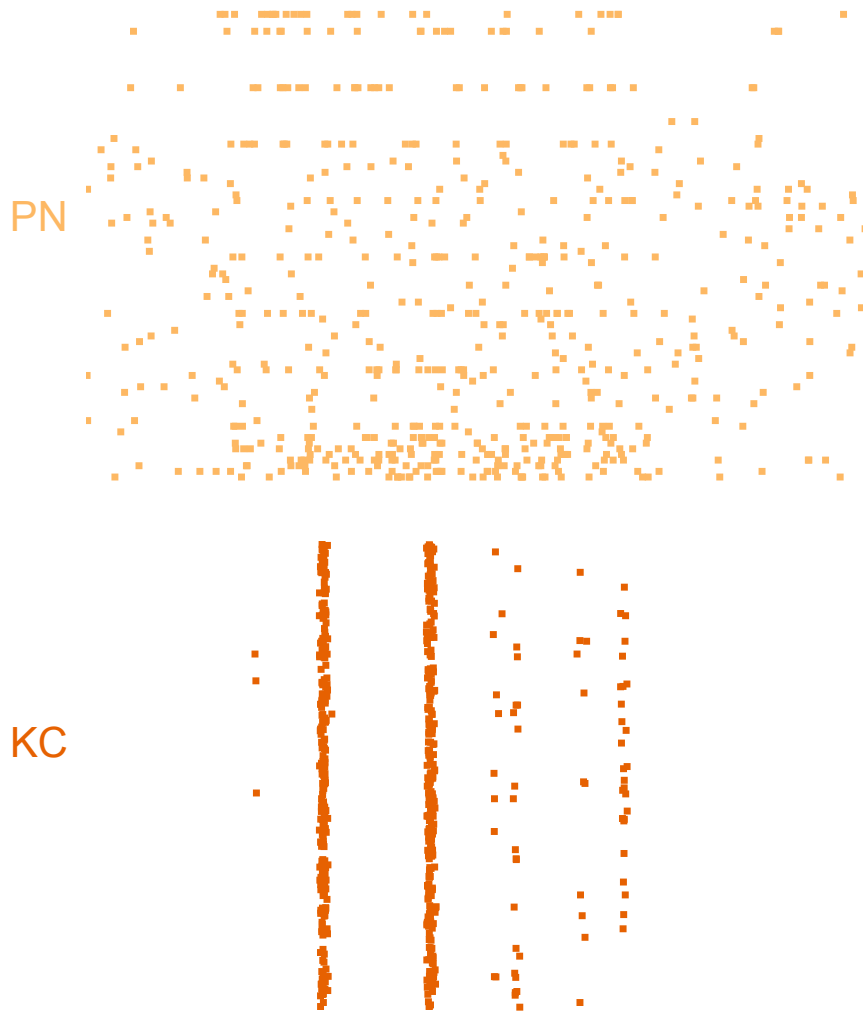

$-40-$

$-45-$

$-50-$

GGN

$-55-$

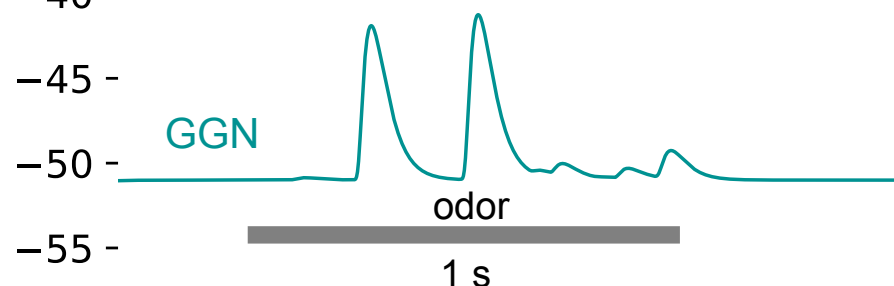

odor

$1 \mathrm{~s}$

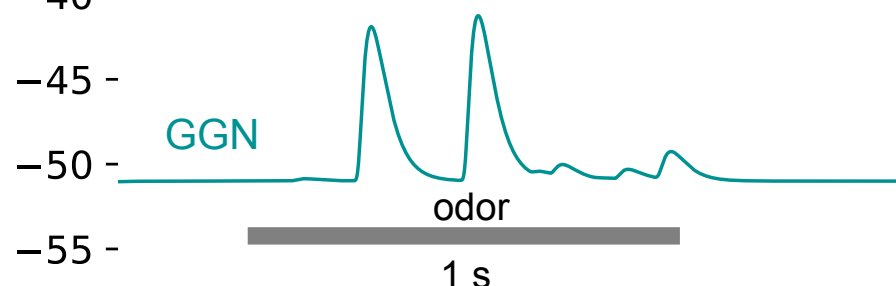

" = 
Figure 5 - figure supplement 2

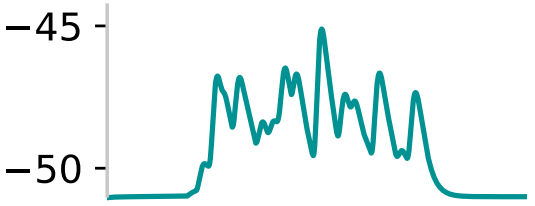

odor 


\section{Figure 6}

a

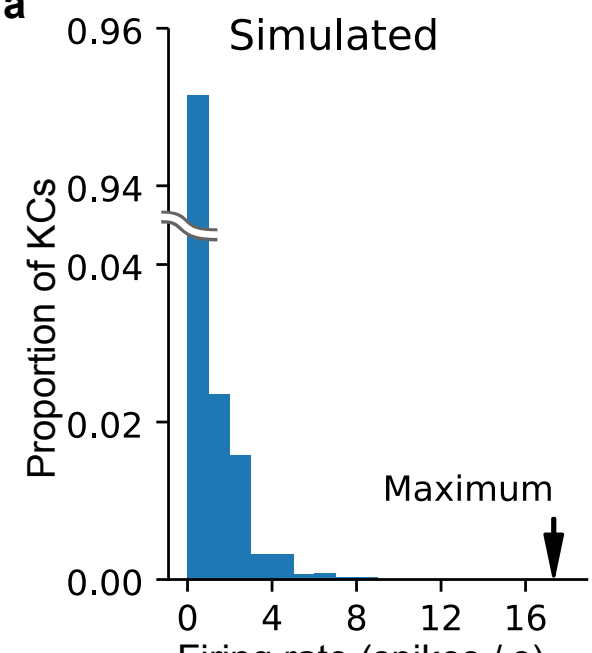

Firing rate (spikes / s)

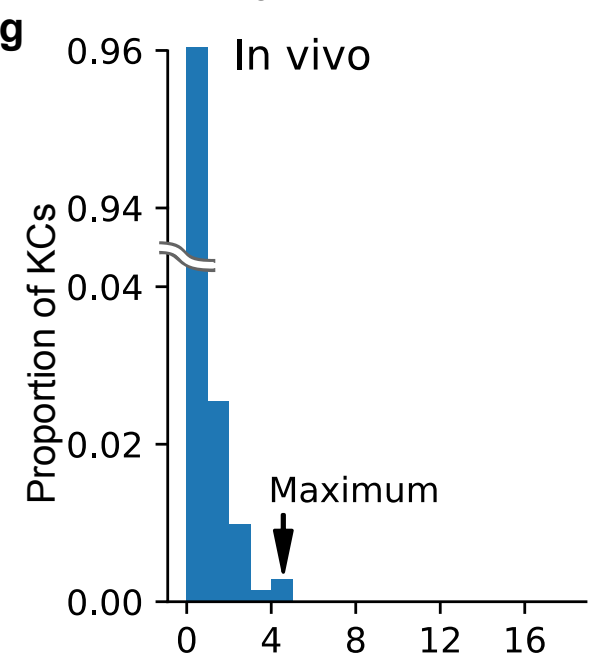

Firing rate (spikes / s) b

b 0.2
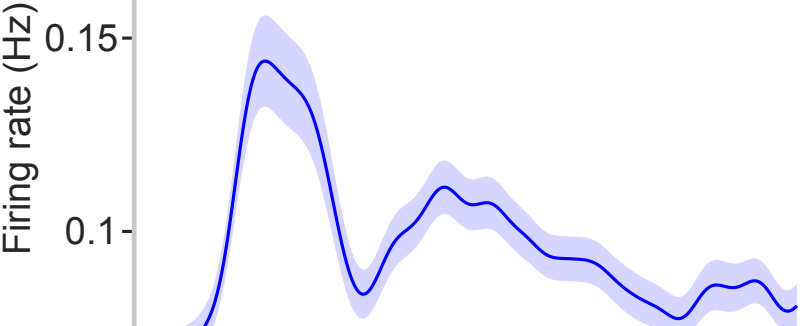

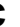

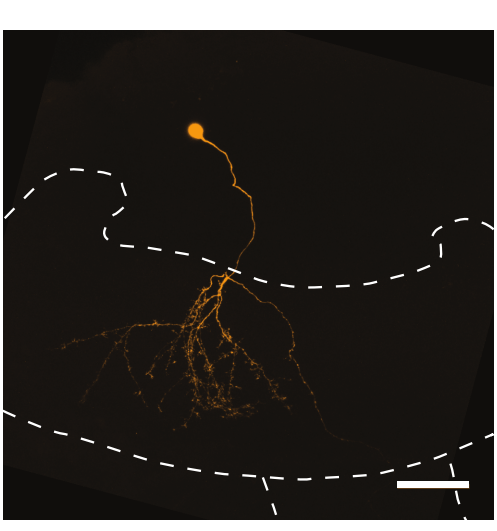

odor

$1 \mathrm{~s}$

f

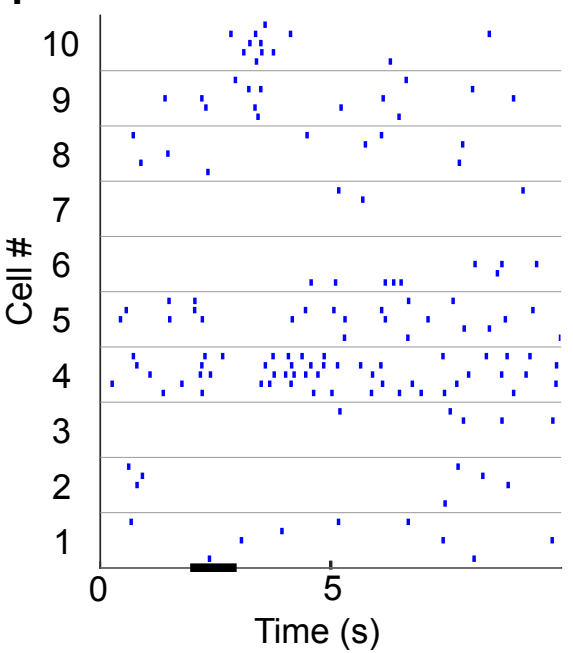

d
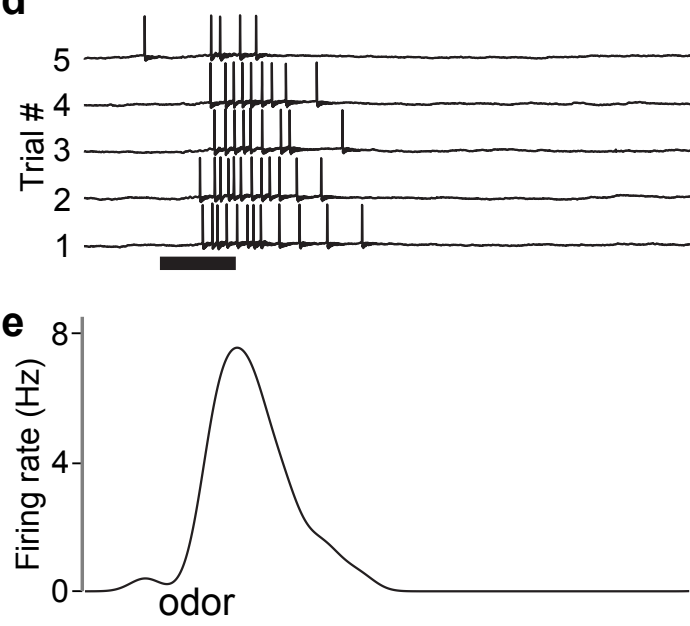

$1 s$ 


\section{Figure 6 - figure supplement 1}
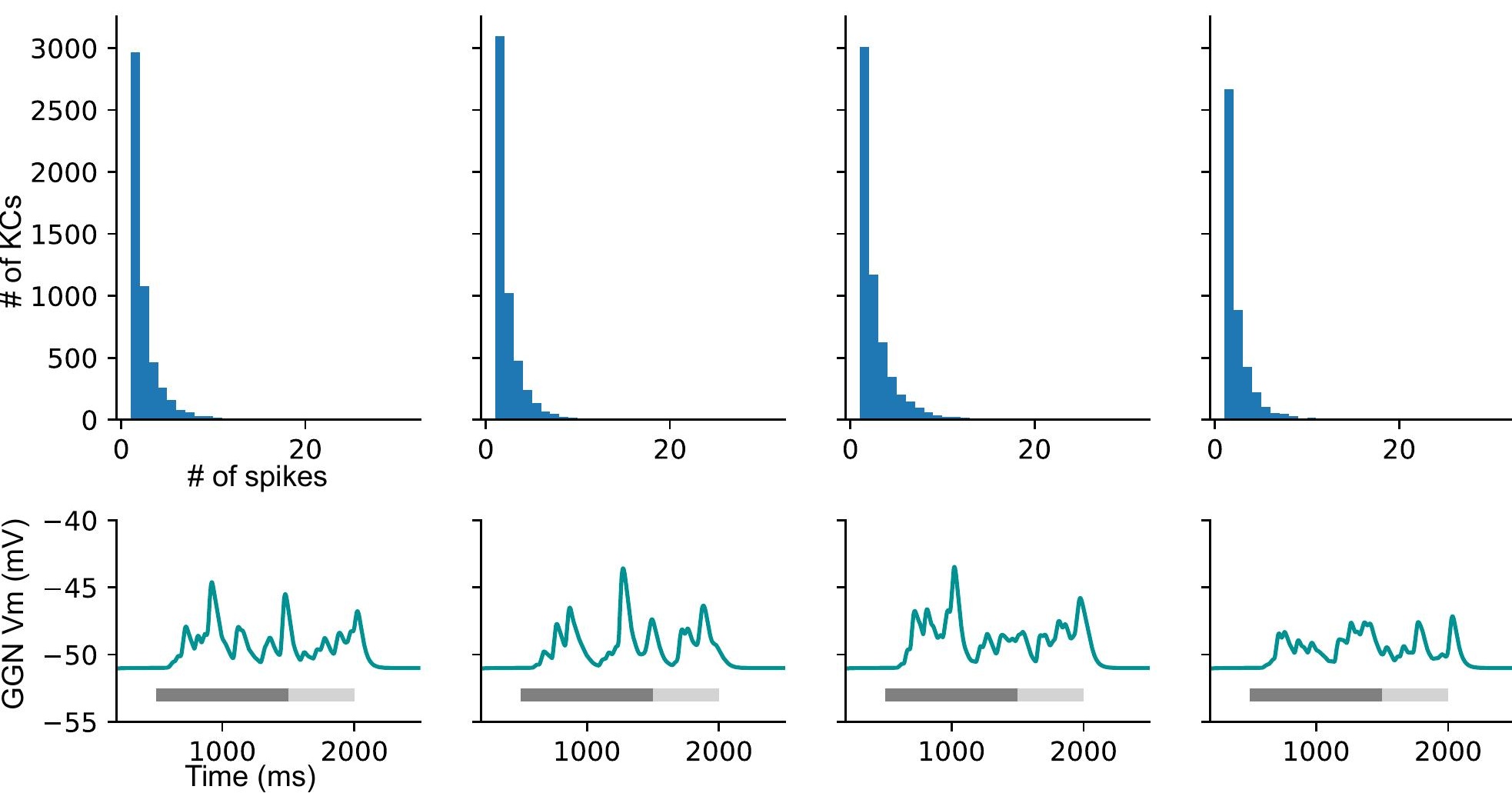
a

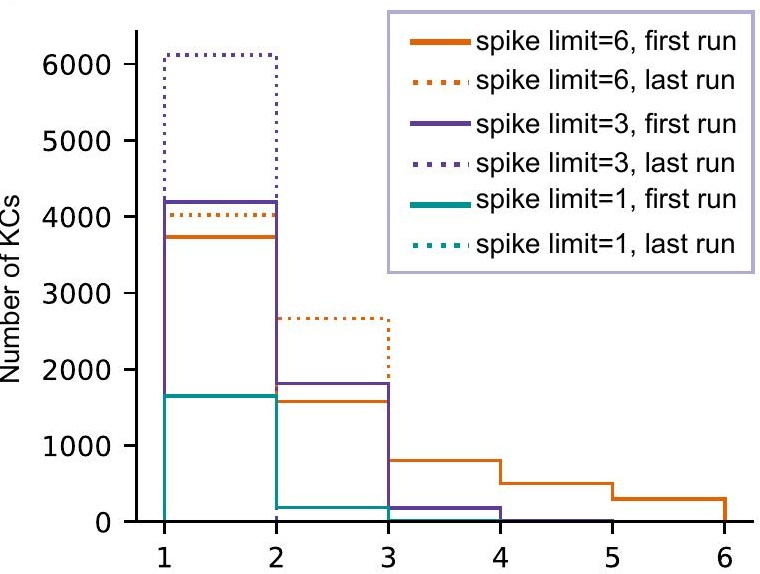

Number of spikes

b

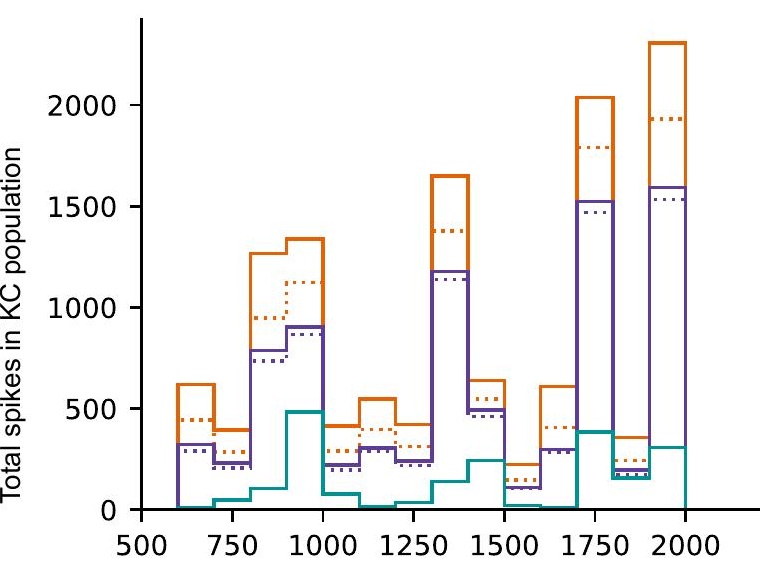

C

:

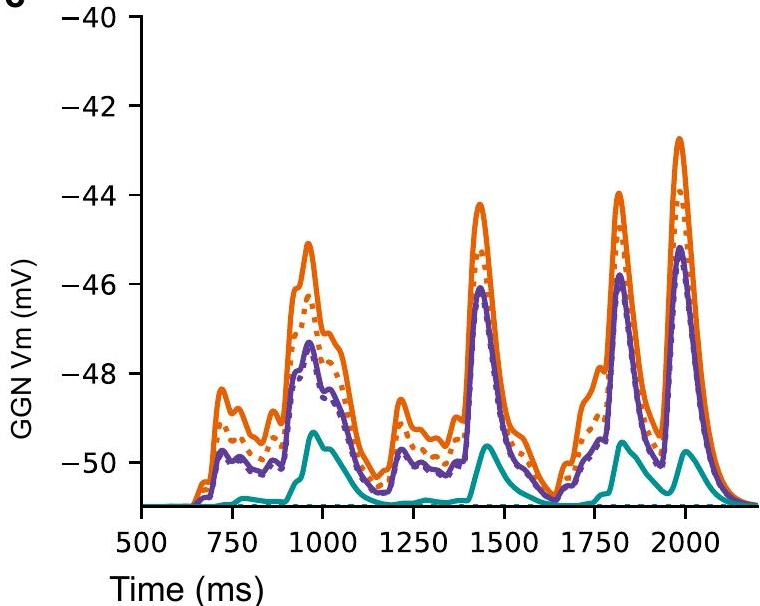


Figure 7 - figure supplement 1

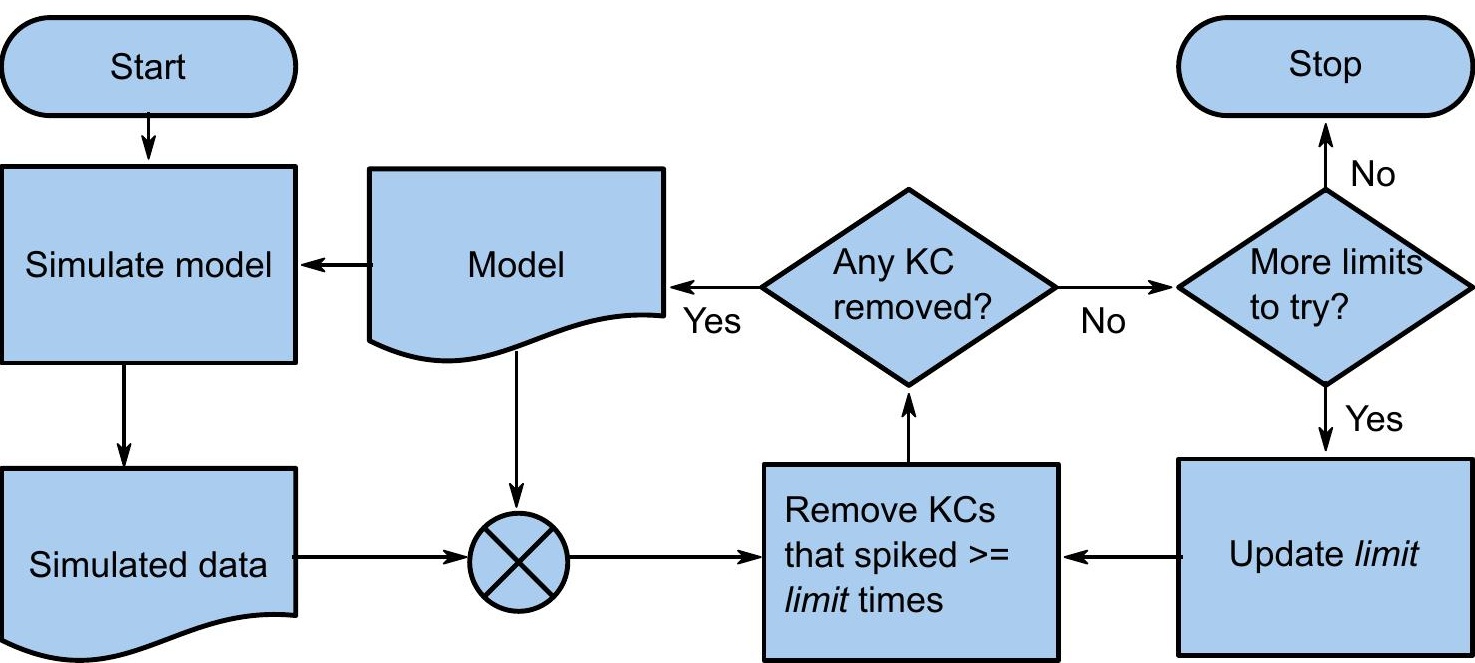


a

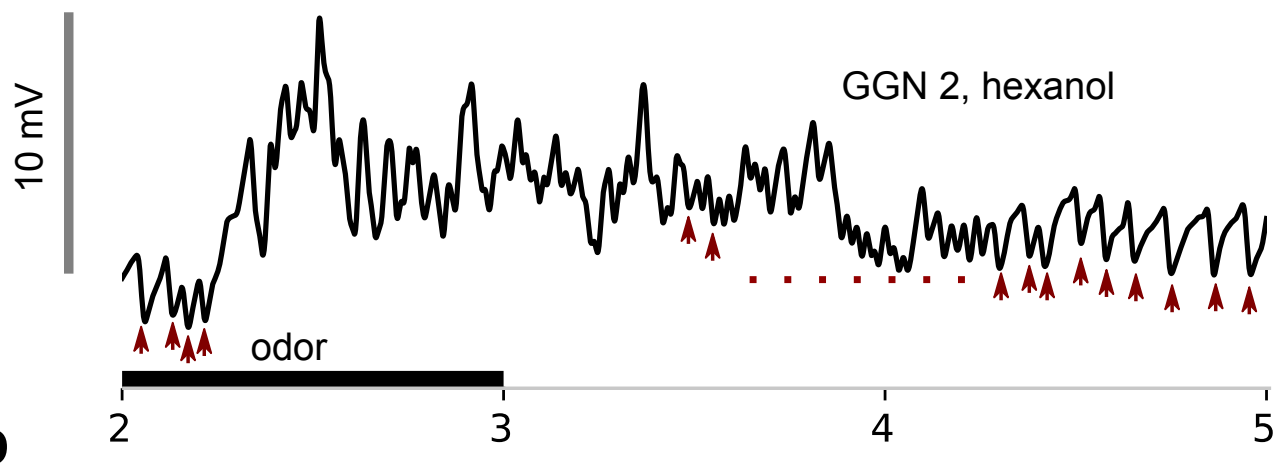

GGN 1, hexanal

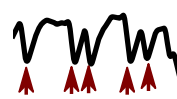

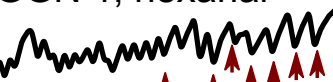

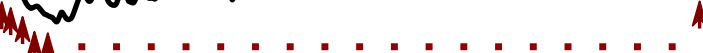

GGN 2, hexanol

.

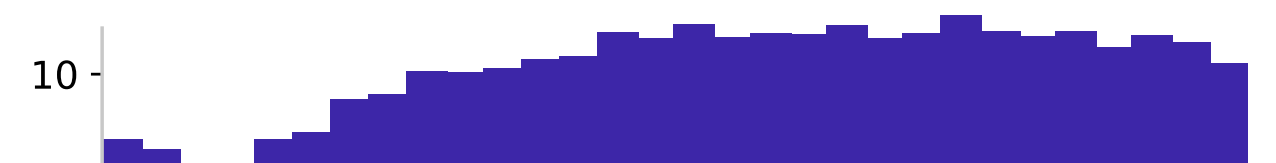

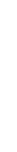

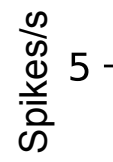

IG PSTH (zoomed)

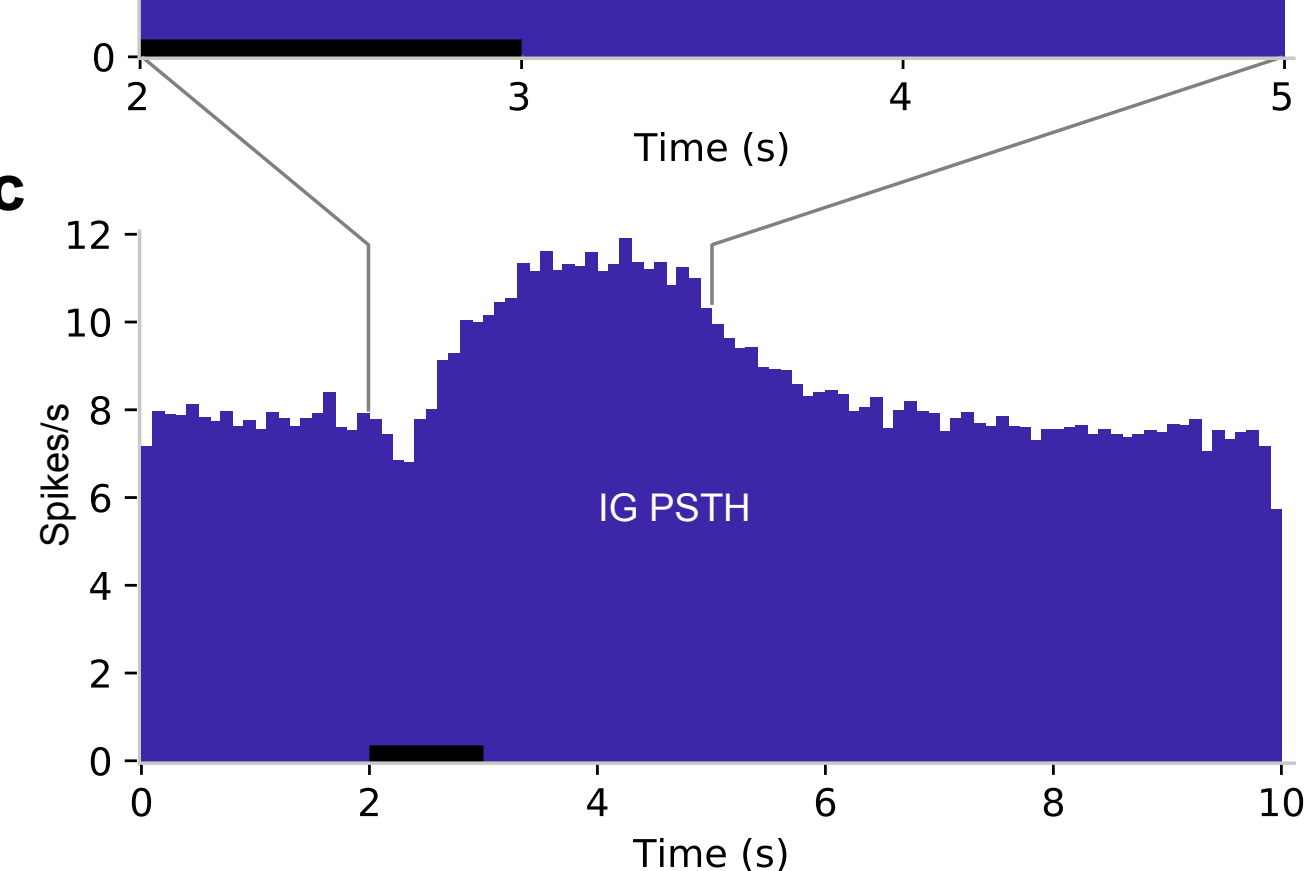


a PNs 030300080300000 j
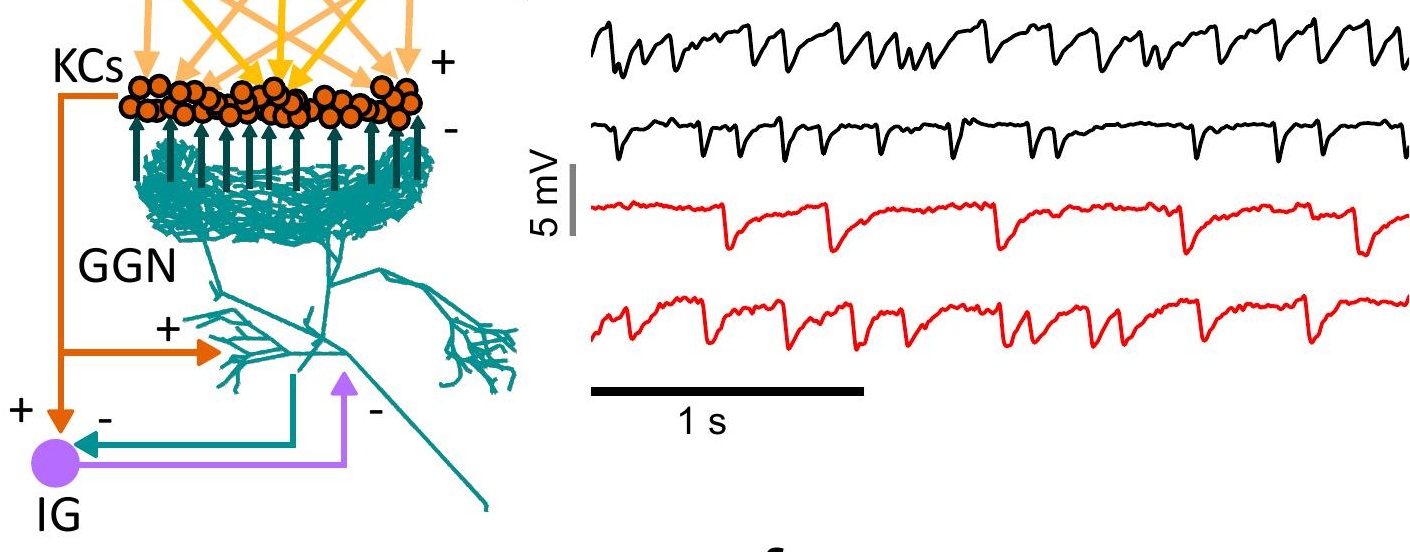

f

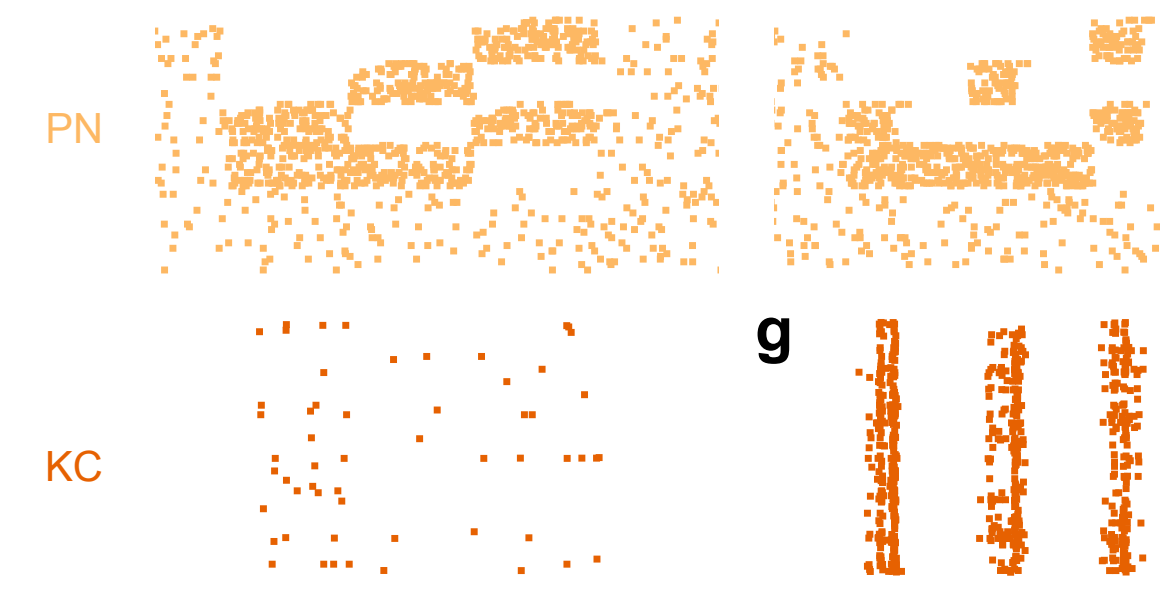

d

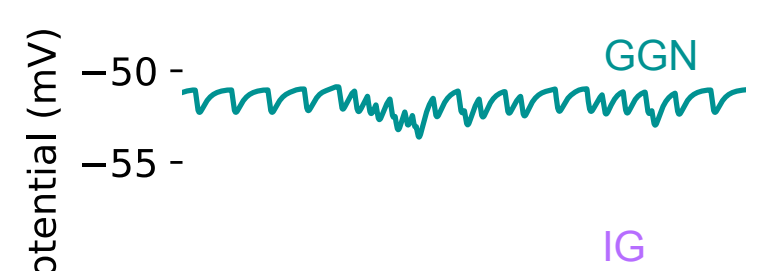

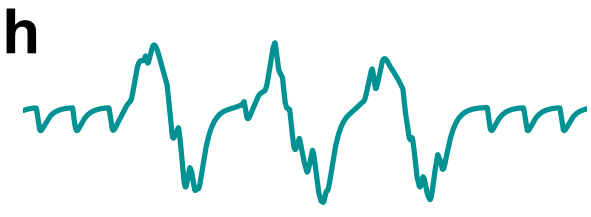

임
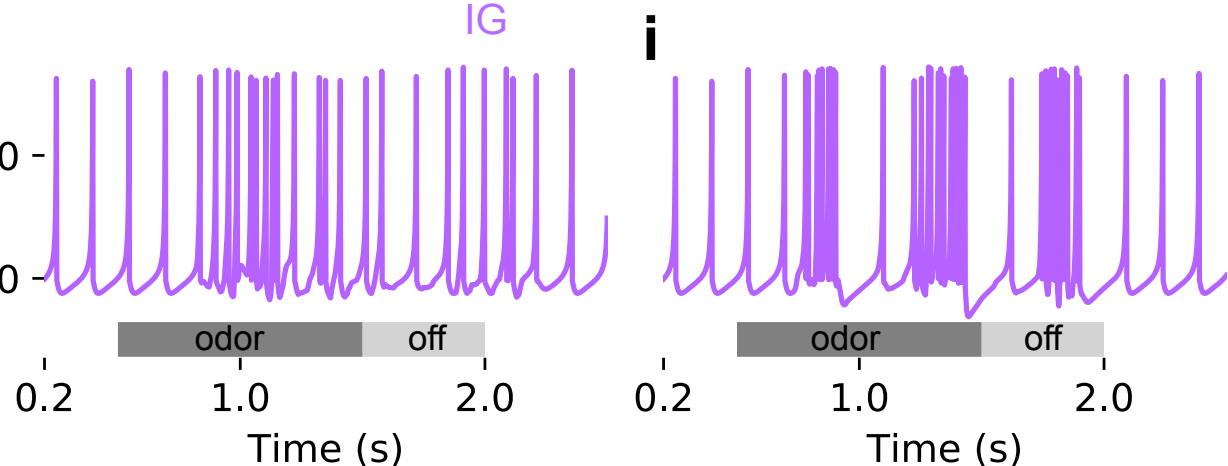

0.2 odor off 1.0 2.0 Time (s) 


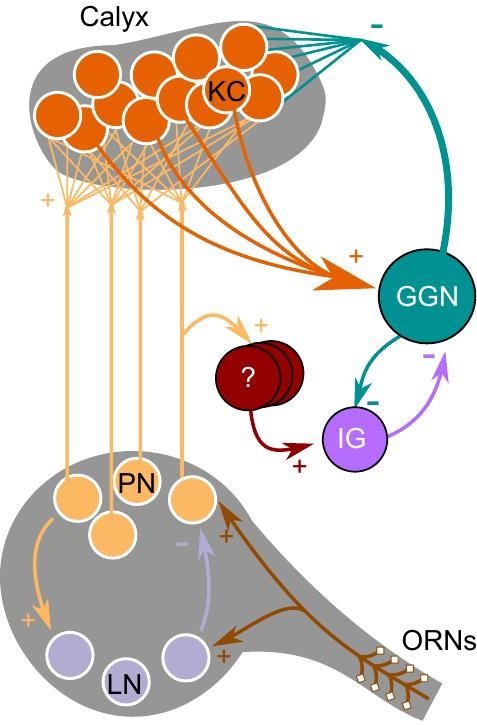

Antennal lobe Antenna 